1

2

3

4

$5{ }^{1}$ School of Biological and Chemical Sciences. Queen Mary University of London. Mile End

6 Road, E1 4NS, London. United Kingdom.

7

$8 \quad{ }^{2}$ These authors contributed equally to this work.

9

10 *Correspondence: chema.martin@qmul.ac.uk

\section{ERK1/2 is an ancestral organising signal in spiral cleavage}

12

\title{
SUMMARY
}

Embryonic organisers are signalling centres that instruct the establishment of body plans during animal embryogenesis, thus underpinning animal morphological diversity. In spiral cleavage - a stereotypic developmental programme ancestral to 14 , nearly half, of the major animal groups (e.g., molluscs, annelids and flatworms), a cell known as the D-quadrant organiser defines cell fates and the body axes. ERK1/2 specifies the embryonic organiser in molluscs, yet how this signalling cascade exerts organising activity and whether this role is conserved in other spiral cleaving groups is unclear. Here, we demonstrate that ERK1/2 promotes the specification and inductive activity of the D-quadrant organiser in Owenia fusiformis, an early-branching annelid exhibiting ancestral developmental traits. In this species, active di-phosphorylated ERK1/2 mediated by FGF receptor activity localises to the $4 \mathrm{~d}$ micromere, establishing the bilateral symmetry and specifying the hindgut and trunk mesodermal progenitor. Accordingly, impairing FGFR and ERK1/2 activity, as well as cell communication results in embryos developing anteroventrally radialised. Differential 
26 transcriptomic profiling shows the ParaHox $c d x$ and the Notch ligand delta as FGFR/ERK1/2

27 downstream targets in $4 \mathrm{~d}$, further revealing that $4 \mathrm{~d}$ specification instructs the expression of

28 mesodermal and posterodorsal genes in neighbouring cells, putatively via the Notch pathway.

29 The instructing role of ERK1/2 in the D-quadrant organiser is thus shared between $O$.

30 fusiformis and molluscs, representing an ancestral trait of spiral cleavage. Altogether, our

31 study begins to dissect the gene network promoting axial patterning and posterior growth in

32 spiral cleavage, revealing extensive mechanistic diversification in body plan specification

33 despite overall conservation of cleavage patterns in Spiralia.

34

35 Keywords: spiral cleavage, Spiralia, embryonic organiser, D-quadrant organiser, annelid,

36 ERK1/2, MAPK pathway, FGF pathway, Notch pathway. 


\section{Introduction}

38 Embryonic organisers are cells or groups of cells able to instruct neighbouring tissues towards certain fates and behaviours during animal embryogenesis [1, 2] (Figure 1A). Embryonic organisers thus play a critical role in patterning tissues and organs, promoting morphogenetic

41 processes that establish animal body plans, thereby emerging as potential drivers of

42 morphological evolution [3-5]. Spiralia is a clade of bilaterally symmetrical animals

43 (Bilateria) comprising nearly half of the major animal clades, including morphologically

44 disparate groups such as molluscs, flatworms and annelids [6, 7]. A stereotypic early

45 developmental programme termed spiral cleavage, characterised by alternating clockwise and

46 counterclockwise oblique cell divisions from the 4-cell stage onwards $[8,9]$ is however

47 widespread, and likely ancestral to Spiralia [8, 10, 11] (Figure 1A). How spiral cleavage generates the vast diversity of spiralian body plans is still unclear (Figure 1A), albeit this being fundamental to understand the evolution of animal development and morphology.

51 Spiral cleavage involves the formation of an embryonic organiser that instructs cell fates around four embryonic quadrants, named A to D, which roughly correspond to the left, ventral, right and dorsal sides of the embryo respectively $[8,9]$. It is a cell belonging to the D54 quadrant - e.g., the 3D or 4d blastomeres in molluscs [12] - that acts as embryonic organiser, and thus this signalling centre is named D-quadrant organiser (Figure 1A). The most common and probably ancestral mode to specify the D-quadrant and its organising cell is through

57 inductive signals at around the 32-cell stage $[13,14]$. This type of spiral cleavage is termed conditional; or equal, since early embryos exhibit quadri-radial symmetry during early stages. However, in some mollusc and annelid species, the specification of the D-quadrant organiser is largely cell-autonomous, as two asymmetric zygotic divisions segregate maternal mRNAs

61 to one (the D) blastomere at the 4-cell stage $[9,13,14]$. This autonomous mode of spiral 
62 cleavage is also referred to as unequal, because the $\mathrm{D}$ founder cell at the 4-cell stage is larger.

63 Regardless of the specification mode, the ERK1/2 signalling pathway, an evolutionarily

64 conserved intracellular cascade of kinases involved in an array of cellular processes [15], is

65 active and necessary in the D-quadrant organiser in molluscs [16-20] (Table S1). However,

66 what activates ERK1/2 and how ERK1/2 orchestrates the instructing role of the D-quadrant

67 organiser in this group is not understood (Figure 1A), and thus how embryonic organisers

68 define the spiralian body plan is still an unresolved question.

69

70 The role of ERK1/2 signalling during spiral cleavage in groups other than Mollusca is in

addition ambiguous and only explored in Annelida (Table S1). As in molluscs, the conditional cleaving annelid Hydroides hexagonus exhibits active ERK1/2 in the 4d cell [17], which is often called "mesentoblast" and cell lineage analyses identify as the trunk mesodermal precursor in most annelid embryos [21-23]. Yet the role of ERK1/2 in the $4 \mathrm{~d}$ cell of $H$. hexagonus is unknown. In autonomous cleaving annelids, the patterns of ERK1/2 activity differ markedly between species (Table S1), but in general ERK1/2 is not active in $4 \mathrm{~d}$ and its inhibition does not result in axial polarity defects in any of the studied species [24-27]. Indeed, the organiser activity occurs in the $2 \mathrm{~d}$ blastomere and $2 \mathrm{~d}^{11}+4 \mathrm{~d}$ in the autonomous cleaving annelids Capitella teleta and Tubifex tubifex respectively [26, 28]; and its inductive role relies on Activin/Nodal activity, and not on ERK1/2 signalling in the autonomous species C. teleta and Chaetopterus pergamentaceus $[24,29]$. Therefore, the cellular and molecular basis underpinning D-quadrant organiser specification and activity varies considerably within Annelida, which ultimately prevents inferring whether ERK1/2 activity in the D-quadrant organiser is a homologous trait to Spiralia or a molluscan innovation that also appeared convergently in some annelid lineages. 
87 The annelid worm Owenia fusiformis belongs to Palaeoannelida - the sister group to all

88 remaining annelids [30] - and exhibits embryonic traits considered ancestral to Annelida,

89 including conditional spiral cleavage, formation of a coeloblastula, gastrulation by invagination and the development of a planktotrophic trochophore-like larva [31] (Figure 1B,

$91 \mathrm{C})$. While inductive signals appear to control the establishment of the axial polarity in $O$.

92 fusiformis [32], which blastomere acts as D-quadrant organiser and how this signalling centre

93 becomes specified and controls body patterning is unknown. Therefore, investigating the

94 nature of the D-quadrant organiser and the role of the ERK1/2 signalling pathway in $O$.

95 fusiformis can, by comparison with other annelid and molluscan lineages, help to infer ancestral character states to Annelida and Spiralia as a whole, ultimately contributing to our understanding of the evolution of spiral cleavage and spiralian body plans.

\section{Results}

\section{Di-phosphorylated ERK1/2 is enriched in the $4 d$ cell in O. fusiformis}

101 The annelid $O$. fusiformis has a single ERK1/2 ortholog (Figure S1A), which is expressed at low levels in active oocytes (Figure S1B). The expression of erkl/2 is stable during early cleavage divisions and up to 4 hours post fertilisation (hpf; 32 cell stage), increasing thereafter and plateauing at the larval stage (Figure S1B). A cross-reactive antibody against the active di-phosphorylated form of ERK1/2 (di-P-ERK1/2) [16, 17] shows weak ubiquitous signal in the zygote and up to $3 \mathrm{hpf}$ ( $\sim 16$ cell stage) (Figure S1C). At $4 \mathrm{hpf}$, however, the four 
112 (Figure 1E). This creates an asymmetry in the timing and order of appearance of the 4q

113 micromeres that is also observed during cleavage of the $3 q$ micromeres, which divide in a

114 counterclockwise order after all 3Q macromeres have cleaved (Figure 1E). By 5 hpf however,

115 the vegetal pole exhibits again a symmetrical arrangement (Figure 1E). At 6 hpf, with the

116 onset of gastrulation [31], di-P-ERK1/2 signal is enriched in seven vegetal cells forming a

117 bilaterally symmetrical pattern that includes one $4 \mathrm{q}$ micromere and the $2 \mathrm{q}^{12}$ and $2 \mathrm{q}^{22}$

118 blastomeres of the other three embryonic quadrants (Figure 1D, Figure S1C). At this stage,

119 the 4Q macromeres have cleaved synchronously into the 5Q and 5q cells and all but the 4q

120 micromere enriched in di-P-ERK1/2 have divided, becoming the earliest morphological sign

121 of bilateral symmetry in $O$. fusiformis embryos. This di-P-ERK1/2 positive 4q micromere

122 divides into two large cells only after ingression during early gastrulation at $7 \mathrm{hpf}$ (Figure 1E),

123 with the later progeny occupying a bilateral position on the posterodorsal side of the

124 archenteron once gastrulation completes at $9 \mathrm{hpf}$ (Figure 1E). The behaviour of this $4 \mathrm{q}$

125 micromere resembles the cellular dynamics described for the $4 \mathrm{~d}$ micromere in other

126 conditional spiral cleaving annelids [33-36], suggesting that the di-P-ERK1/2 enriched 4q

127 micromere at 5 and $6 \mathrm{hpf}$ is the $4 \mathrm{~d}$ cell and its two large daughter cells are the MR/ML

128 mesoteloblasts (Figure 1D, E). Therefore, ERK1/2 signalling activates first in the $1 \mathrm{q}^{111}$ animal

129 micromeres and subsequently in the $4 \mathrm{~d}$ micromere in the annelid $O$. fusiformis, which is

130 similar to the spatiotemporal dynamics of ERK1/2 activity reported in spiral cleaving

131 molluscs (Table S1).

132

ERK1/2 signalling controls axial polarity in O. fusiformis

134 To examine the role of the ERK1/2 signalling during $O$. fusiformis development, we treated

135 embryos with brefeldin A (BFA), an inhibitor of intracellular protein trafficking previously

136 used to block the induction of the organiser in other spiral cleaving embryos [32,37], and 
137 U0126, a selective inhibitor of MEK1/2 and ERK1/2 di-phosphorylation [16, 38] (Figure 2A).

138 For both drugs, treatment from fertilisation ( $~ 0.5 \mathrm{hpf})$ to $5 \mathrm{hpf}$, when di-P-ERK1/2 is enriched 139 in 4d, effectively blocks activation of ERK1/2 (Figure 2B; Figures S2A; Table S2) and causes

140 the loss of bilateral symmetry, posterior structures (e.g., chaetae and hindgut) and larval

141 muscles in $100 \%$ of the embryos at a $10 \mu \mathrm{M}$ concentration (Figure 2C; Figure S2B; Table S3,

142 S4). Compared to control samples, 0.5 to 5 hpf treated embryos lack a fully formed apical tuft

143 and apical organ, showing reduced ectodermal expression of the apical organ marker six3/6

144 [39] and just a few apical cells positive for the neuronal marker synaptotagmin-1 (syt-1) [31]

145 (Figure 2D). In addition, treated embryos lack expression of hindgut $(c d x)$ and trunk

146 mesodermal (twist) markers [39], exhibit expanded expression of the oral ectodermal marker

147 gene gsc [39] around the single gut opening (Figure 2D; Figure S2C), and retain expression of

148 the midgut endodermal marker GATA4/5/6b [39] (Figure S2C). We deem this phenotype as

149 antero-ventrally radialised (or Radial; Figure 2C, E). Therefore, activation of ERK1/2

150 signalling in the $4 \mathrm{~d}$ cell at the coeloblastula stage relies on inductive cell-cell communication

151 signals (impaired by BFA treatment) required to specify and develop posterior and dorsal

152 structures during $O$. fusiformis embryogenesis.

153

154 To dissect the exact timing of induction and activity of ERK1/2 during O. fusiformis cleavage,

155 we treated embryos with $10 \mu \mathrm{M} \mathrm{BFA} / \mathrm{U} 0126$ in overlapping time windows from fertilisation

156 to early gastrulation (Figure 2E; Table S5). Blocking protein secretion with BFA from

157 fertilisation to the 8-cell stage does not affect normal development. However, BFA treatment

158 between the 8-cell stage and 4 hpf results in larvae with all morphological landmarks of a

159 typical mitraria larva and normal expression of tissue-specific markers, but with a compressed

160 morphology (Figure 2F; Figure S2C). Only treatment with BFA from 4 hpf to 6 hpf, and

161 hence spanning the formation of $4 \mathrm{~d}$, causes a radial phenotype, with treatments after $5 \mathrm{hpf}$ 
162 being lethal (Figure 2E, Figure S2C). This suggests that the intercellular communication event

163 inducing the activation of ERK1/2 in 4d happens between 4 and 5 hpf, right during 4q

164 micromere formation (Figure 1E). Unexpectedly, preventing ERK1/2 di-phosphorylation with

165 U0126 from the 2-cell stage until 4 hpf, when this induction event might begin, also causes a

166 radial phenotype (Figure 2E), with just slight differences between certain timepoints; embryos

167 treated between 2-cells and $3 \mathrm{hpf}$ show expression of the mesodermal marker twist in a few

168 discrete cells, and embryos treated from 4 to $6 \mathrm{hpf}$ are seemingly more elongated along the

169 apical-oral axis (Figure S2C). Therefore, ERK1/2 activity is essential for normal embryonic

170 patterning and posterodorsal development throughout most spiral cleavage in $O$. fusiformis.

171 However, the combination of BFA and U0126 phenotypes suggest that ERK1/2 acts

172 autonomously from the 2-cell stage to $4 \mathrm{hpf}$, while it requires of inductive cell-to-cell

173 communication signals for its enrichment in $4 \mathrm{~d}$.

175 ERK1/2 signalling activates posterodorsal and mesodermal genes

176 To investigate the mechanisms through which ERK1/2 controls posterodorsal development in

177 O. fusiformis, we hypothesised that genome-wide profiling of gene expression in BFA and

178 U0126 treated embryos would uncover upstream regulators and downstream targets of

179 ERK1/2 activity. We thus treated embryos with either $10 \mu \mathrm{M}$ BFA or $10 \mu \mathrm{M}$ U0126 from

180 fertilisation to 5 hpf (to cover both the autonomous and conditional phases of ERK1/2

181 activity) and performed RNA-seq transcriptome profiling in treated and controlled embryos

182 collected right after di-P-ERK1/2 enrichment in the 4d cell (coeloblastula; $5.5 \mathrm{hpf}$ ) and at the

183 larval stage (Figure 3A). Principal component analysis (PCA) and sample-to-sample distance

184 matrices clustered transcriptomic profiles according to developmental stage and biological

185 replica (Figure S3A, B). Differential expression analyses revealed 90 and 268 differentially

186 expressed genes (DEGs; Log Fold Change < -1.5 and adjusted p-value < 0.05 ) in BFA treated 
187 coeloblastula and larva respectively, and 132 (coeloblastula) and 373 (larva) DEGs after

188 U0126 treatment (Figure 3B). When considering all comparisons and removing redundancies, 189 we detected a total of 628 DEGs, 414 (65.92\%) of which were functionally annotated and 190 enriched in gene ontology terms related to regulation of transcription, development and cell

191 fate specification (Table S6, S7, S8). Most of these DEGs were downregulated (Figure 3B, C;

192 Figure S3C, D), with 26 genes downregulated under both drug treatments at the coeloblastula 193 stage and 154 genes commonly downregulated at the larval stage. Only three DEGs (cdx, fer3 194 and $f o x H$ ) appeared systematically downregulated in both drugs and in the two developmental 195 time points, while we did not identify any common upregulated DEG (Figure 3B; Figure 196 S3D). Therefore, our approach revealed a confident and relatively small set of genes whose 197 expression is dependent on ERK1/2 activity and early inductive signals in $O$. fusiformis 198 embryos.

200 To validate the set of genes affected by $4 \mathrm{~d}$ misspecification, we selected 22 candidate genes

201 for further gene expression analyses (Figure 3D, Table S9), including a variety of

202 transcription factors recurrently involved in axial embryonic patterning (e.g., six3/6, gsc, $c d x$,

203 AP2, foxQ2), mesoderm development (e.g., twist, hand2, foxH) and neurogenesis (e.g., POU4,

204 irxA), Wnt ligands (wnt1, wntA and wnt4), TGF- $\beta$ modulators (noggin and BAMBI), and

205 Notch signalling components (delta and notch-like) (Table S8). Stage-specific RNA-seq data

206 covering twelve developmental time-points, from the unfertilized oocyte to the mature larva,

207 confirmed that for all candidate genes, their expression upregulates at the time of or just after

$2084 d$ specification and di-P-ERK1/2 enrichment in this cell (Figure 3D). These genes are 209 expressed either in apical/anterior domains (noggin, BAMBI, foxQ2, POU4, six3/6, gsc), the

210 posterior larval tip and chaetae (fer3, lhx1/5, wnt1, notch, msx2-a, irxA, AP2, wnt4, delta), the 211 hindgut ( $c d x)$, or mesodermal derivatives (foxH, rhox, wntA, POU3, hand2, twist) (Figure 3D, 
212 Figure S4). Analysis of the expression of these genes in control and treated embryos at the

213 coeloblastula (5.5hpf) and larva (24hpf) stages confirmed the expression domains of these

214 genes disappear after treatment with either BFA or U0126 (Figure 3E, Figure S4), thus

215 validating our RNA-seq approach. Altogether, these results define a set of co-regulated genes

216 that act downstream of ERK1/2 signalling and/or early inductive events, and that are involved

217 in the development of apical, posterodorsal and mesodermal structures in $O$. fusiformis.

219 ERK1/2 signalling specifies and patterns the D-quadrant

220 Our RNA-seq study and candidate gene screening revealed nine genes expressed at the vegetal pole at $5.5 \mathrm{hpf}$ and whose expression was affected by either direct inhibition of ERK1/2 di-phosphorylation (cdx, delta, foxH, wnt1, wntA, rhox, fer3 and AP2) or impairing cell-to-cell inductive signals ( $g s c$ ) (Figure 3E, Figure S4). None of these genes are expressed at $5 \mathrm{hpf}$, at the onset of ERK1/2 activity in the $4 \mathrm{~d}$ micromere (Figure 4A). Instead, the early endodermal marker GATA4/5/6a [39], whose expression is unaffected by BFA and U0126 treatment and thus is cell-autonomous, is symmetrically expressed in the gastral plate (including 4d) at this stage (Figure 4A; Figure S5A). At 5.5 hpf, half an hour after the initial activation of ERK1/2 signalling in 4d, the vegetal pole becomes bilaterally symmetrically patterned, but only two of the ERK1/2 dependent genes ( $c d x$ and delta) are expressed in the $4 \mathrm{~d}$ micromere (Figure 4A). The ParaHox gene $c d x$, which is detected in the hindgut of $O$.

231 fusiformis at the larval stage [39] (Figure 3E; Figure S5B), becomes expressed in 4d, and later 232 on in two cells of the MR and ML progeny at the gastrula stage (Figure 4B), as expected from the behaviour of $4 \mathrm{~d}$ at these stages (Figure 1E). Similarly, the Notch ligand delta (Figure S5C, D) is expressed in $4 \mathrm{~d}$ at $5.5 \mathrm{hpf}$, but also in most of the descendants of $1 \mathrm{~d}$ at the animal pole,

235 plus $1 \mathrm{a}-\mathrm{c}^{1121}$ and ectodermal derivatives of the $\mathrm{C}$ and $\mathrm{D}$ quadrant at the vegetal pole (Figure 
237 for Transposase-Accessible Chromatin using sequencing (ATAC-seq) data at 5 hpf and

238 identified known bound transcription factors in the accessible regions associated with these

239 genes (Figure 4D), which include DNA binding motifs for transcription factors known to be

240 modulated by ERK1/2 phosphorylation, such as ETS, RUNX and GATA factors [15].

241 Therefore, ERK1/2 di-phosphorylation in 4d seems to delay cell cycle progression in this cell

242 with respect to $4 \mathrm{a}-\mathrm{c}$ (Figure 1E) and control the activity of transcriptional regulators that

243 induce posterior fates $(c d x)$ and cell-cell communication genes (delta).

244

245 The other six additional ERK1/2-dependent genes pattern the micromeres surrounding $4 \mathrm{~d}$ at

\section{$5.5 \mathrm{hpf}$, defining mesodermal and posterior ectodermal domains (Figure 4A). The}

transcription factor foxH (Figure S5E), which regulates mesoderm development during gastrulation in vertebrate embryos [40-43], is detected in the $3 \mathrm{c}^{22}$ and $3 \mathrm{~d}^{22}$ micromeres adjacent to 4d (Figure 4A), whose progeny contributes to lateral ectomesoderm in the annelid Urechis caupo [36]. Later in development, foxH is expressed in a posterior V-shaped pattern of putative mesodermal precursors during axial elongation, fading away in larval stages (Figure S5B). The ligands wnt1 and wntA (Figure S5), expressed in the posterior region and D-quadrant during development in the annelid Platynereis dumerilii [44], are expressed in two bilaterally symmetrical columns of micromeres $\left(3 c^{22}, 3 c^{21}, 3 c^{12}, 3 c^{11}, 3 d^{22}, 3 d^{21}, 3 d^{12}\right.$, $3 \mathrm{~d}^{11}$ ) plus the micromeres $2 \mathrm{~d}^{222}$ and $2 \mathrm{~d}^{112}$ in $w n t A$ (Figure $4 \mathrm{~A}$ ). The homeobox rhox (Figure $\mathrm{S} 5 \mathrm{G})$, which is expressed in male and female primordial germ cells in vertebrates [45], is detected in the micromeres $2 \mathrm{~d}^{112}$ and $2 \mathrm{~d}^{212}$ (Figure 4A) and thereafter in two small cells inside the blastocele in the gastrula (Figure S4). The transcription factors fer 3 and $A P 2$ are expressed in two single micromeres $\left(1 \mathrm{c}^{222}\right.$ and $\left.1 \mathrm{~d}^{221}\right)$ and in a broader posterior ectodermal domain $\left(1 \mathrm{c}^{222}, 1 \mathrm{~d}^{221}, 2 \mathrm{~d}^{122}, 2 \mathrm{~d}^{212}, 2 \mathrm{~d}^{2111}, 2 \mathrm{~d}^{2112}, 2 \mathrm{~d}^{1112}, 2 \mathrm{~d}^{1111}\right)$ respectively, becoming restricted to a small expression domain at or near the posterior chaetal sac of the larva (Figure S4; Figure 
262 S5B). The larger size of $4 \mathrm{~d}$, which stays as a big posterior cell at $5.5 \mathrm{hpf}$, allows it to establish

263 almost as double direct cell-cell contacts with its surrounding cells than each of the daughter

264 cells of $4 \mathrm{a}-\mathrm{c}$, including most of the cells expressing foxH, wnt1, wntA, rhox, and AP2 (Figure

265 4E). This data demonstrates the inductive role of the $4 \mathrm{~d}$ in defining mesodermal and

266 posterodorsal fates, and together with the upregulation of the Notch-ligand delta in $4 \mathrm{~d}$ after

267 ERK1/2 activation, suggests that this role might occur by direct cell-cell communication

268 mediated by the Notch signalling pathway.

270 The homeobox gene $g s c$ is the only candidate expressed outside the D-quadrant at $5.5 \mathrm{hpf}$,

271 being detected in micromeres $2 \mathrm{a}^{11}-2 \mathrm{c}^{11}, 2 \mathrm{a}^{12}-2 \mathrm{c}^{12}, 2 \mathrm{a}^{21}-2 \mathrm{c}^{21}$, and $2 \mathrm{a}^{22}-2 \mathrm{c}^{22}$ (Figure $4 \mathrm{~A}$ ).

272 Consistent with its location outside the D-quadrant, gsc expression is independent of ERK1/2

273 activity, but requires of inductive signals, as demonstrated by being downregulated after BFA

274 treatment (Table S3). Accordingly, gsc expression disappears in BFA-treated coeloblastulae,

275 while inhibition of ERK1/2 activity with U0126 expands gsc domains, which becomes

276 detected in all embryonic quadrants in a radial fashion (Figure 4F). Indeed, in ERK1/2

277 inhibited embryos all $4 q$ micromeres cleave into $4 q^{1}$ and $4 q^{2}$, as no $4 q$ becomes the $4 d$ cell

278 (Figure 4F). Therefore, all quadrants adopt antero-ventral fates ( $g s c)$, preventing the

279 expression of posterior marker genes such as AP2 (Figure 4F). These results demonstrate that

280 the radial phenotype observed after U0126 is a consequence of misspecifying the $4 \mathrm{~d}$ cell, and

281 not a result of the D-quadrant becoming specified but not developing further. In addition, this

282 data also demonstrates that specification of the 4d cell through ERK1/2 activity represses

283 anterior fates, as shown by limiting gsc expression. 
285

286

287

288

289

290

291

292

293

294

295

296

297

298

299

300

301

302

303

304

305

306

307

308

309

\section{FGF signalling regulates ERK1/2 di-phosphorylation in $4 d$}

In O. fusiformis, $c d x$ and delta are expressed in 4d under regulation of ERK1/2, and their expression is associated with hindgut and trunk mesoderm development, as well as posterior growth (Figure 2C). In vertebrate embryos, FGF signalling mediated by ERK1/2 activity regulates the expression of $c d x$ and delta in the developing hindgut and presomitic mesoderm during posterior elongation [46] and FGF signalling promotes mesoderm development in the spiralian groups Brachiopoda and Phoronida [47]. We thus hypothesised that FGF signalling might be the upstream regulator driving ERK1/2 activity in 4d (Figure 5A). O. fusiformis has a single FGF receptor (FGFR; Figure S6A) with high amino acid conservation at key functional residues compared to the human FGFR1 ortholog (Figure 6B). While it is transcriptionally upregulated at the gastrula stage, FGFR appears weakly expressed at the gastral plate at the coeloblastula stage in O. fusiformis (Figure S6C, D), as observed in brachiopods and phoronids [47]. At this stage, FGF ligands and VEGFR, the other tyrosine kinase receptor inhibited by SU5402, are only weakly expressed (Figure S6C) and not detected by in situ hybridisation (Figure S6D). Treatment with $30 \mu \mathrm{M}$ SU5402, a selective inhibitor of FGFR phosphorylation and activation [48] (Figure 5A), prevents di-P-ERK1/2 enrichment at $4 \mathrm{~d}$ cell in $96 \%$ of the treated embryos at 5 hpf (Figure 5B, Figure S6E, Table S2). Indeed, treatment with SU5402 during the specification of the $4 \mathrm{~d}$ micromere (4 to $6 \mathrm{hpf}$ and 3 to 7 hpf; Figure 5C) results in an anteriorly radialised phenotype, with embryos developing into larvae lacking posterior and reduced mesodermal structures and showing radially expended oral ectodermal fates ( $g s c$ ) (Figure 5D, Table S3). Conversely, treatment before di-P-ERK1/2 enrichment in $4 \mathrm{~d}$ (3 to $5 \mathrm{hpf}$ ) causes a slightly compressed phenotype (Figure S6F). Therefore, inhibition of FGFR blocks ERK1/2 activation and phenocopies the effect of U0126 and BFA (Figure 2C), suggesting that FGF signalling activity is upstream of and necessary for $4 \mathrm{~d}$ specification in $O$. fusiformis. 


\section{Discussion}

312 Our study provides compelling evidence that FGFR and ERK1/2 signalling specify and

313 control the organising activity of the $4 \mathrm{~d}$ micromere in the conditional spiral cleaving annelid

314 O. fusiformis (Figure 6A). FGFR/ERK1/2 signalling at around $5 \mathrm{hpf}$ is necessary for

315 instructing one of the recently emerged $4 \mathrm{q}$ micromeres into the $\mathrm{D}$ fate (Figure $2 \mathrm{E}$, Figure $5 \mathrm{C}$,

316 Figure 6A). However, 4q micromeres form already asymmetrically at $4 \mathrm{hpf}$ in $O$. fusiformis

317 (Figure 1E), yet whether there is an exact correspondence between the order of formation and

318 the fate of each $4 \mathrm{q}$ remains elusive. Asynchronies in the cleavage of 3Q have not been

319 described for other conditional cleaving annelids [33-35], but are more common in molluscs,

320 where $4 \mathrm{~d}$ can be either the first or the last micromere to form $[3,12,14]$. This asynchrony in

$3213 \mathrm{Q}$ cleavage, together with the fact that ERK1/2 signalling affects axial polarity

322 independently of cell communication (i.e., unaffected by BFA) earlier than 4 hpf (Figure 2E,

323 Figure S2C) suggest that cell-autonomous ERK1/2-dependent mechanisms might act before

324 the inductive specification of $4 \mathrm{~d}$ in $O$. fusiformis. In other systems, MAPK controls unequal

325 protein segregation during mitosis via pericentrosomal material [49], which is the subcellular

326 region where maternal mRNAs condense before asymmetric inheritance during spiral

327 cleavage in the mollusc Tritia obsoleta [50], and MAPK controls mRNA stability during early

328 zygotic divisions in the annelid Helobdella robusta [51]. Therefore, basal levels of ERK1/2

329 signalling during early cleavage might control the activity or differential segregation of

330 determinants required to respond adequately to the inductive signals specifying the $4 \mathrm{~d}$ cell in

331 O. fusiformis. Although further work is needed to test this scenario, our findings indicate that

332 both autonomous and conditional mechanisms are essential to specify the D-quadrant

333 organiser in O. fusiformis, challenging traditional views that conditional/equal spiral cleaving

334 species rely mostly on inductive signals to establish their body pattern. 
336 The specification of $4 \mathrm{~d}$ micromere has at least four immediate consequences during $O$.

337 fusiformis embryogenesis (Figure 6A). First, and contrary to its role in promoting cell

338 proliferation [15], ERK1/2 activity correlates with 4d cell cycle arrest, breaking the quatri-

339 radial symmetry of the embryo as the $4 \mathrm{a}-4 \mathrm{c}$ micromeres cleave (Figure 1E; Figure 6A). In

340 some molluscs, activation of ERK1/2 in the 3D blastomere also correlates with cleavage

341 delays $[12,17]$, and thus a potential role of ERK1/2 signalling in arresting, instead of

342 promoting, cell division appears to be common in the context of the embryonic organiser in

343 spiralian embryos. Second, specification of $4 d$ and the D-quadrant represses anterior fates to

344 the A-C quadrants, as observed by the expression of the BFA-affected oral ectodermal gene

$345 g s c$ in all four quadrants in ERK1/2 inhibited embryos (Figure 4F). These findings suggest

346 that two as yet unknown opposing signals - one specifying anterior fates and promoting gsc

347 expression, and another driving 4d specification via FGFR/ERK1/2 - might act

348 simultaneously at 4-5 hpf (Figure 6A). Considering that gsc expression is eventually

349 recovered in BFA treated larvae (Figure S2C), we hypothesise that this anteriorising signal

350 must come from cell lineages already specified by $4-5 \mathrm{hpf}$, such as the endoderm/midgut

351 (Figure 4A) or the apical organ (Figure 2D). Third, FGF/ERK1/2 activity upregulates the

352 hindgut gene $c d x$ in 4d (Figure 3C; Figure 4A; Figure 6A). Inhibition of both FGF and

353 ERK1/2 activity affects hindgut but not foregut and midgut development (Figure 2C; Figure

354 5D), indicating that unlike most annelids [21] but similar to molluscs [3, 52], 4d contributes to

355 both endoderm/hindgut and mesoderm in O. fusiformis. Fourth, FGF/ERK1/2 activity also

356 upregulates the Notch ligand delta in $4 \mathrm{~d}$, as well as a set of mesodermal and posterior genes,

357 Wnt ligands and TGF- $\beta$ modulators in its surrounding cells, most of which establish a direct

358 cell-to-cell contact with 4d (Figure 4A, E; Figure 6A). Therefore, 4d instructs cell fates in the

359 D and C quadrant at the vegetal pole, probably through the Notch signalling pathway, as also 
360 proposed for the mollusc Tritia obsoleta [53]. Altogether, our study demonstrates the

361 organising properties of the $4 \mathrm{~d}$ cell (endomesodermal precursor) in $O$. fusiformis, defining a

362 comprehensive, testable model to study the establishment of the body plan in this and other

363 spiral cleaving organisms.

364

365 Enrichment of di-phosphorylated ERK1/2 in the 4d micromere is common to O. fusiformis

366 and H. hexagonus, the two conditional spiral cleaving annelids studied so far [17], which

367 contrasts with the variability in ERK1/2 activity observed among autonomous cleaving annelids [25-27]. Likewise, the 4d cellular identity of the D-quadrant organiser in $O$. fusiformis differs from the autonomous annelid species C. teleta and T. tubifex, which concentrate the organising activity in the $2 \mathrm{~d}$ and $2 \mathrm{~d}^{11}+4 \mathrm{~d}$ cells respectively $[26,28]$.

371 However, the $4 \mathrm{~d}$ cell identity observed in $O$. fusiformis resembles the molluscan condition,

372 where ERK1/2 is active in 3D and maintained in 4d, both of which can exert organising 373 activity $[9,12]$. Moreover, $c d x$ and delta, the downstream targets of ERK1/2 in the $4 \mathrm{~d}$ of $O$.

374 fusiformis, are also expressed in $4 \mathrm{~d}$ in the mollusc Tritia obsoleta [52, 53], further suggesting

375 conservation in both signalling cascades and transcriptional outputs between D-quadrant

376 organisers in annelids and molluscs. Therefore, our study supports that ERK1/2 activity in the

377 cell acting as embryonic organiser is a shared homologous trait between Annelida and

378 Mollusca and given our current understanding of Spiralia phylogeny [6, 7], a probable

379 ancestral character to at least the internal node Lophotrochozoa. Under this scenario,

380 heterochronic shifts to confer organising activity to $2 \mathrm{~d}$ micromeres as seen in C. teleta and $T$.

381 tubifex $[26,28]$ and the use of Activin/Nodal signalling instead of ERK1/2 to establish the body plan $[24,29]$ are thus derived conditions. 
384 More broadly, our study uncovers a role of the FGF signalling pathway, an evolutionarily

385 conserved upstream regulator of mesoderm development in bilaterians $[47,54,55]$, in the

386 specification of 4d, the D-quadrant organiser and endomesodermal precursor in $O$. fusiformis

387 (Figure 5B-D). FGFR activity in $4 \mathrm{~d}$ results in the activation of $c d x$, Notch signalling (delta)

388 and Wnt signalling (wnt1, wntA) in vegetal micromeres of the D-quadrant (Figure 4A) that

389 will occupy the posterodorsal blastoporal lip as gastrulation begins, supporting that

390 blastopore-associated axial organisers also occur during spiral cleavage [4]. Furthermore,

391 FGF, Wnt and Notch signalling, together with $c d x$ are all conserved gene modules regulating

392 posterior axis elongation in an array of bilaterian embryos (Figure 6B) [39, 46, 47, 56-58].

393 Classically, axial elongation in spiralian embryos has been proposed to occur by proliferation

394 of the $2 \mathrm{~d}$ clone $[59,60]$, which express delta and wntA in O. fusiformis (Figure 4A), further

395 suggesting that specification of $4 \mathrm{~d}$ also results in the deployment of broadly conserved genetic

396 mechanisms involved in posterior growth that eventually promote axial (and blastopore)

397 elongation in O. fusiformis (Figure 2C; Figure 6B). Altogether, our study demonstrates that

398 the establishment of the axial identities (A-D quadrants), endomesoderm specification,

399 posterodorsal patterning and axial elongation are all intimately connected events that depend

400 on the instruction of a single cell fate (the $4 \mathrm{~d}$ cell) and use evolutionarily conserved

401 mechanisms in O. fusiformis, a conditional spiral cleaving annelid. Decoupling these

402 embryonic organising processes through maternal determinants (e.g., as in autonomous spiral

403 cleavers), heterochronic shifts in organiser specification and/or signalling pathway rewiring

404 (e.g., co-option of Activin/Nodal) might have thus contributed to the emergence of the vast

405 diversity of body plans observed today in Spiralia.

406

407 Acknowledgements 
408 We thank the staff at Station Biologique de Roscoff for their help with collections and animal

409 supplies, as well as the Oxford Genomics Centre at the Wellcome Centre for Human Genetics

410 (funded by Wellcome Trust grant reference 203141/Z/16/Z) for the generation and initial

411 processing of RNA-seq and ATAC-seq sequencing data. We thank all members of the Martín-

412 Durán lab for support and valuable comments on the manuscript. This work was funded by a

413 European Research Council Starting Grant (action number 801669) to J.M.M.-D. and a Queen

414 Mary, University of London studentship to O.S.

415

416 Author Contributions

417 O.S., A.M.C.-B. and J.M.M.-D. conceived and designed the study. O.S., A.M.C.-B., Y.L. and

418 J.M.M.-D. performed all experimental approaches and critically analysed the data. O.S.,

419 A.M.C.-B. and J.M.M.-D. wrote the manuscript. All authors read and commented on the

420 manuscript.

421

\section{Declaration of Interests}

423 The authors declare no competing interests. 


\section{Materials and Methods}

\section{Animal husbandry and embryo collections}

426 Adult specimens of $O$. fusiformis Delle Chiaje, 1844 were collected from the coast near the

427 Station Biologique de Roscoff (France) during the reproductive season (May to July). In the

$428 \mathrm{lab}$, animals were kept in artificial seawater (ASW) at $15^{\circ} \mathrm{C}$. In vitro fertilizations were

429 conducted as previously described [31, 39] and embryos develop in glass bowls with filtered

$430 \mathrm{ASW}$ at $19^{\circ} \mathrm{C}$ until the desired embryonic stage.

\section{Drug treatments}

433 Embryos were treated with either brefeldin A (BFA; Sigma-Aldrich, \#B7651),

434 U0126 (Sigma-Aldrich, \#U120), or SU5402 (Sigma-Aldrich, \#572630). All drug stocks were

435 made in dimethyl sulfoxide (DMSO) and diluted in ASW prior use, with optimal working

436 concentrations (10 $\mu \mathrm{M}$ for BFA and U0126 and $30 \mu \mathrm{M}$ for SU5402) established after initial

437 titration. For all drug treatments, equivalent volumes of DMSO were used as negative control,

438 and in all cases, treatments were initiated after $\sim 0.5$ hours post fertilisation (hpf), right after

439 fertilisation and sperm removal. Treatments were stopped by washing out the drug with at

440 least three washes in ASW and embryos were either collected for downstream analyses or

441 raised until larval stage. Samples collected for immunohistochemistry and gene expression

442 analyses were fixed in 4\% paraformaldehyde (PFA) in ASW for $1 \mathrm{~h}$ at room temperature (RT).

443 Larvae were relaxed in 8\% magnesium chloride $\left(\mathrm{MgCl}_{2}\right)$ prior fixation and fixed in $4 \%$ PFA

444 in $8 \% \mathrm{MgCl}_{2}$ for $1 \mathrm{~h}$ at $\mathrm{RT}$. After fixation, samples were washed several times with $0.1 \%$

445 Tween-20 phosphate-buffered saline (PTw), and either stored in PTw at $4^{\circ} \mathrm{C}$ for

446 immunohistochemistry or dehydrated to $100 \%$ methanol and stored at $-20^{\circ} \mathrm{C}$ for whole mount

447 in situ hybridisation. Treated and control embryos and larvae collected for RNA-seq analyses

448 were snap frozen in liquid nitrogen and stored at $-80^{\circ} \mathrm{C}$ prior total RNA extraction. 
$450 \quad$ Immunohistochemistry

451 F-actin labelling, and antibody staining were conducted as previously described [31], with the

452 modification that samples collected for anti-di-P-ERK1/2 staining were washed in PTw

453 supplemented with 1:100 dilution of phosphatase inhibitors (Cell Signalling, \#5872) after

454 fixation. The primary antibodies mouse anti-double phosphorylated ERK1/2 (Sigma-Aldrich,

455 \#M8159, 1:200) and mouse anti-acetylated $\alpha$-tubulin (clone 6-11B-1, Millipore-446 Sigma,

456 \#MABT868, 1:500) were diluted in 5\% normal goat serum (NGS) in $0.1 \%$ Triton X-100

457 phosphate-buffered saline (PTx) and incubated overnight $(\mathrm{ON})$ at $4{ }^{\circ} \mathrm{C}$. After several washes

458 in $1 \%$ bovine serum albumin (BSA) in PTx, samples were incubated with either an anti-

459 mouse peroxidase (POD) conjugated antibody (Millipore-Sigma, \#11207733910, 1:100) or an

460 AlexaFluor594 conjugated antibody (ThermoFisher Scientific, \#A32731) diluted in 5\% NGS

461 in PTx ON at $4^{\circ} \mathrm{C}$. For samples incubated with mouse anti-double phosphorylated ERK1/2

462 and anti-mouse POD conjugated antibody, signal was developed using either

463 diaminobenzidine (DAB) (Vector Laboratories, \#SK-4100) or Tyramide Signal Amplification

464 (TSA) kit (Akoya Biosciences, \#NEL742001KT) following manufacturer's

465 recommendations. DAB-developed samples were stored in $70 \%$ glycerol and counterstained

466 with 4',6-diamidino-2- phenylindole (DAPI, ThermoFisher Scientific, \#D3571, 1:1000)

467 nuclear marker. Samples for laser scanning confocal microscopy (LSCM) were

468 counterstained with DAPI diluted in $1 \%$ bovine serum albumin (BSA) in PTx for $1 \mathrm{~h}$ at RT

469 and mounted in $70 \%$ glycerol.

470

471 Gene isolation and gene expression analyses

472 Candidate genes were amplified by two rounds of nested PCR using gene specific primers and

473 a T7 universal primer on cDNA from mixed developmental stages as initial template (Table 
474 S9). Riboprobes were synthesized with the T7 enzyme (Ambion's MEGAscript

475 kit, \#AM1334) and stored in hybridisation buffer at a concentration of $50 \mathrm{ng} / \mu \mathrm{l}$.

476 Colorimetric and fluorescent whole-mount in situ hybridisation were performed following an

477 established protocol [31, 39]. After probe washes, samples for fluorescent in situ

478 hybridisation were incubated with anti-DIG-POD Fab fragments (ROCHE, \#11633716001)

479 and a mouse anti-acetylated $\alpha$-tubulin antibody (clone 6-11B-1, Millipore-446 Sigma,

480 \#MABT868, 1:500) to co-stain cell boundaries. After signal development with the TSA kit

481 (Akoya Biosciences, \#NEL742001KT), these samples were washed several times in PTx and

482 treated for secondary antibody incubation as described above.

483

484

Genome sequencing, gene annotation and RNA-seq developmental time course

High molecular weight genomic DNA was obtained from a single $O$. fusiformis male

following the agar plug extraction method and sequenced on a PacBio Sequel II platform to a

200x coverage. Initial assembly was conducted with Canu [61], subsequently scaffolded

with BioNano optical mapping data generated on an Irys platform and finally de-haploidised

with purge_haplotigs [62]. To annotate protein coding genes, we used available

transcriptomic data (SRA accession number SRR1222288) and generated replicated stage-

491

specific RNA-seq data covering twelve developmental time-points, from the unfertilized

492

active oocyte until the mature mitraria larva. RNA-seq reads were mapped with STAR [63]

493

and gene models were generated combining Mikado v.2.1 [64], Augustus [65] and PASA [66]

494 pipelines. Gene completeness was assessed with BUSCO [67] (97.5\% complete, 1.5\%

495

fragmented, $1 \%$ missing). Trinotate [68] and Panther [69] were used to functionally annotate 
499

500

501

502

503

504

505

506

507

508

509

510

511

512

513

514

515

516

517

518

519

520

521

522

523

available in $\mathrm{R}$, were colour intensity shows the $\mathrm{z}$-score value for each gene. All raw sequencing data is available under the project with accession number $\mathrm{XXXXXX}$ in the European Nucleotide Archive (ENA).

\section{Assay for Transposase-Accessible Chromatin using sequencing (ATAC-seq)}

Two replicated ATAC-seq libraries were generated from $>50,000$ cells at $5 \mathrm{hpf}(850-900$ coeloblastulae) following the Omni-ATAC protocol [70], employing a home-made Tn5 transposase [71] and amplifying for 12 cycles. Libraries were sequenced on an Illumina Hiseq4000 platform, 75 base paired-end output mode. After adaptor removal with cutadapt v.2.5 [72], reads were mapped with NextGenMap v.0.5.5 [73] and duplicates removed with samtools v.1.9 [74]. BAM files were shifted using deepTools2 v.3.4.3 [75] and MACS2 v2.2.7.1 $[76,77]$ was used for peak calling with parameters -f BAMPE --min-length 100 -max-gap 75 -q 0.01. Reproducible peaks $(\mathrm{p}<0.05)$ were identified applying Irreproducible Discovery Rate package v2.0.4.2 [78]. Transcription factor motif enrichment was performed with HOMER v.4.11 [79] and transcription factor foot printing with TOBIAS v.0.12.0 [80]. To identify bound transcription factors on peaks of $c d x$ and delta, we intersected the genomic positions of peaks and bound transcription factors with BEDTools v.2.29.2 [81]. Genomic tracks were plotted with pyGenomeTracks v.2.1 [82].

\section{RNA-seq profiling and differential gene expression analyses}

Total RNA was extracted using the Monarch total RNA Miniprep Kit (New England Biolabs, \#T2010) and used for Illumina stranded mRNA library prep. Sequencing was performed on an Illumina HiSeq 4000 platform in 75 bases paired-end output mode, generating 20 million reads per sample. Adapter sequences and low-quality bases were removed using Trimmomatic v.0.36 [83]. Cleaned reads were then mapped to $O$. 
524 fusiformis reference genome annotation using Kallisto v.0.44.0 to generate gene expression

525 counts [84]. Differential gene expression analyses were performed independently for each

526 drug and computed in pairwise comparisons of different stages and conditions using the $\mathrm{R}$

527 package DEseq2 v.1.38.0 [85]. Significancy threshold was adjusted to a p-value equal or

528 lower than 0.05 and a $\log 2$ fold change greater than 1.5 or lower than -1.5 [86]. PCAs and

529 hierarchical clustering were plotted using ggplot2 and pheatmap R packages,

530 respectively [87]. Volcano plots were obtained with the package EnhancedVolcano, available

531 in R. Candidate genes for further gene expression analyses were selected based on being

532 differentially expressed in both drug treatments (U0126 and BFA) or highly differentially

533 expressed at $5.5 \mathrm{hpf}$ in U0126 treated embryos. We further refined our selection based on

534 gene ontology (GO) categories, focusing on transcription factors and developmental genes

535 (Table S7).

536

537 GO and KEGG enrichment analyses

538 For GO mapping, the GO terms of differentially expressed genes (DEGs) associated with

539 each comparison were extracted from $O$. fusiformis reference genome annotation. GO

540 enrichment analyses were implemented using the GOseq R package, correcting for gene

541 length bias [88], and analysing up-regulated and down-regulated genes independently. GO

542 terms with corrected p-values lower than 0.05 were considered to be significantly enriched

543 (Table S7). A similar approach was followed for Kyoto Encyclopaedia of Genes and

544 Genomes (KEGG) pathway enrichment analyses, mapping differentially represented KO

545 numbers to their respective KEGG pathway [89] (Table S8).

546 


\section{Orthology assignment and domain architecture analyses}

548 Generally, gene orthology was based on the functional annotation of $O$. fusiformis gene

549 repertoire, based on BLAST searches against SwissProt database and Panther searches [69,

550 90]. Protein sequences for ERK1/2 and ERK5, WNT ligands, RHOX and related

551 homeoboxes, DLL and JAGGED, FOXH and FOXF genes and FGFR and VEGFR were

552 retrieved by mining publicly available transcriptomes and databases. Multiple protein

553 alignments (Supplementary File 1) were constructed with MAFFT v.7 in automatic mode [91]

554 and poorly aligned regions were removed with gBlocks version 0.91b [92]. Maximum

555 likelihood trees were constructed with either RAxML v.8.2.11 [93] or FastTree [94] and

556 visualised with FigTree (https://github.com/rambaut/figtree/). InterProScan 5 [95] was used to

557 perform the protein domain composition of DLL.

558

$559 \quad$ Imaging

560 Representative embryos and larvae from colorimetric analyses were imaged with a Leica

561 DMRA2 upright epifluorescent microscope equipped with an Infinity5 camera (Lumenera),

562 using bright field and differential interference contrast optics. Fluorescently stained samples

563 were scanned with a Leica SP5 LSCM. Confocal stacks were analysed with Fiji and

564 brightness/contrast and colour balance were adjusted in Pixelmator Pro (v. 2.0.3) or

565 Photoshop (Adobe), applying changes always to the whole image and not parts of it. Final

566 figures were designed using Illustrator (Adobe). 


\section{References}

568 1. Martinez Arias, A., and Steventon, B. (2018). On the nature and function of $569 \quad$ organizers. Development 145.

$570 \quad$ 2. Anderson, C., Khan, M.A.F., Wong, F., Solovieva, T., Oliveira, N.M.M., Baldock,

571 R.A., Tickle, C., Burt, D.W., and Stern, C.D. (2016). A strategy to discover new

572 organizers identifies a putative heart organizer. Nat Commun 7, 12656.

573 3. Lyons, D.C., Perry, K.J., Lesoway, M.P., and Henry, J.Q. (2012). Cleavage pattern

574 and fate map of the mesentoblast, $4 \mathrm{~d}$, in the gastropod Crepidula: a hallmark of

575 spiralian development. Evodevo 3, 21.

576 4. Kraus, Y., Aman, A., Technau, U., and Genikhovich, G. (2016). Pre-bilaterian origin 577 of the blastoporal axial organizer. Nat Commun 7, 11694.

578 5. Lapraz, F., Haillot, E., and Lepage, T. (2015). A deuterostome origin of the Spemann 579 organiser suggested by Nodal and ADMPs functions in Echinoderms. Nat Commun 6 , $580 \quad 8434$.

581 6. Laumer, C.E., Bekkouche, N., Kerbl, A., Goetz, F., Neves, R.C., Sorensen, M.V., Kristensen, R.M., Hejnol, A., Dunn, C.W., Giribet, G., et al. (2015). Spiralian phylogeny informs the evolution of microscopic lineages. Curr Biol 25, 2000-2006.

584 7. Marlétaz, F., Peijnenburg, K., Goto, T., Satoh, N., and Rokhsar, D.S. (2019). A New 585 Spiralian Phylogeny Places the Enigmatic Arrow Worms among Gnathiferans. Curr $586 \quad$ Biol 29, 312-318 e313.

587 8. Martín-Durán, J.M., and Marletaz, F. (2020). Unravelling spiral cleavage. $588 \quad$ Development 147.

589 9. Henry, J.Q. (2014). Spiralian model systems. Int J Dev Biol 58, 389-401.

590 10. Hejnol, A. (2010). A twist in time--the evolution of spiral cleavage in the light of 591 animal phylogeny. Integr Comp Biol 50, 695-706. 
592 11. Schleip, W. (1929). Die Determination der Primitiventwicklung, (Leipzig:

593 Akademische Verlagsgesellschaft).

594 12. Lambert, J.D. (2008). Mesoderm in spiralians: the organizer and the 4d cell. J Exp Zool B Mol Dev Evol 310, 15-23.

596 13. Freeman, G., and Lundelius, J.W. (1992). Evolutionary implications of the mode of D quadrant specification in coelomates with spiral cleavage. Journal of Evolutionary Biology 5, 205-247.

599 14. Van Den Biggelaar, J.A.M., Van Loon, A.E., and Damen, W.G.M. (1995).

600 Mesentoblast and Trochoblast Specification in Species With Spiral Cleavage Predict

601 Their Phyletic Relations. Netherlands Journal of Zoology 46, 8-21.

602

15. Lavoie, H., Gagnon, J., and Therrien, M. (2020). ERK signalling: a master regulator of 603 cell behaviour, life and fate. Nat Rev Mol Cell Biol 21, 607-632.

16. Lambert, J.D., and Nagy, L.M. (2001). MAPK signaling by the D quadrant embryonic organizer of the mollusc Ilyanassa obsoleta. Development 128, 45-56.

606 17. Lambert, J.D., and Nagy, L.M. (2003). The MAPK cascade in equally cleaving 607 spiralian embryos. Dev Biol 263, 231-241.

608

18. Lartillot, N., Lespinet, O., Vervoort, M., and Adoutte, A. (2002). Expression pattern of Brachyury in the mollusc Patella vulgata suggests a conserved role in the establishment of the AP axis in Bilateria. Development 129, 1411-1421.

611 19. Henry, J.J., and Perry, K.J. (2008). MAPK activation and the specification of the D 612 quadrant in the gastropod mollusc, Crepidula fornicata. Dev Biol 313, 181-195.

613 20. Koop, D., Richards, G.S., Wanninger, A., Gunter, H.M., and Degnan, B.M. (2007). 614 The role of MAPK signaling in patterning and establishing axial symmetry in the gastropod Haliotis asinina. Dev Biol 311, 200-212. 
616 21. Ozpolat, B.D., Handberg-Thorsager, M., Vervoort, M., and Balavoine, G. (2017). Cell

617 lineage and cell cycling analyses of the $4 \mathrm{~d}$ micromere using live imaging in the marine $618 \quad$ annelid Platynereis dumerilii. Elife 6.

619 22. Gline, S.E., Nakamoto, A., Cho, S.J., Chi, C., and Weisblat, D.A. (2011). Lineage 620 analysis of micromere 4d, a super-phylotypic cell for Lophotrochozoa, in the leech $621 \quad$ Helobdella and the sludgeworm Tubifex. Dev Biol 353, 120-133.

622 23. Meyer, N.P., Boyle, M.J., Martindale, M.Q., and Seaver, E.C. (2010). A

623 comprehensive fate map by intracellular injection of identified blastomeres in the 624 marine polychaete Capitella teleta. Evodevo 1, 8.

625 24. Lanza, A.R., and Seaver, E.C. (2020). Activin/Nodal signaling mediates dorsal-ventral 626 axis formation before third quartet formation in embryos of the annelid Chaetopterus pergamentaceus. Evodevo 11, 17.

628 25. Pfeifer, K., Schaub, C., Domsch, K., Dorresteijn, A., and Wolfstetter, G. (2014). Maternal inheritance of twist and analysis of MAPK activation in embryos of the polychaete annelid Platynereis dumerilii. PLoS One 9, e96702.

26. Amiel, A.R., Henry, J.Q., and Seaver, E.C. (2013). An organizing activity is required teleta: new insights into cell-cell signaling in Lophotrochozoa. Dev Biol 379, 107-122.

634 27. Kozin, V.V., Filimonova, D.A., Kupriashova, E.E., and Kostyuchenko, R.P. (2016). Mesoderm patterning and morphogenesis in the polychaete Alitta virens (Spiralia, Annelida): Expression of mesodermal markers Twist, Mox, Evx and functional role for MAP kinase signaling. Mech Dev 140, 1-11. 
641 29. Lanza, A.R., and Seaver, E.C. (2020). Functional evidence that Activin/Nodal

642 signaling is required for establishing the dorsal-ventral axis in the annelid Capitella

643 teleta. Development 147.

644 30. Weigert, A., Helm, C., Meyer, M., Nickel, B., Arendt, D., Hausdorf, B., Santos, S.R., 645 Halanych, K.M., Purschke, G., Bleidorn, C., et al. (2014). Illuminating the base of the annelid tree using transcriptomics. Mol Biol Evol 31, 1391-1401.

647 31. Carrillo-Baltodano, A.M., Seudre, O., Guynes, K., and Martin-Duran, J.M. (2021). 648 Early embryogenesis and organogenesis in the annelid Owenia fusiformis. Evodevo $649 \quad 12,5$.

650 32. Gonzales, E.E., van der Zee, M., Dictus, W.J., and van den Biggelaar, J. (2007).

651 Brefeldin A and monensin inhibit the D quadrant organizer in the polychaete annelids 652 Arctonoe vittata and Serpula columbiana. Evol Dev 9, 416-431.

653 33. Treadwell, A.L. (1901). The cytogeny of Podarke obscura Verrill. J. Morph. 17, 399$654 \quad 478$.

655 34. Mead, A.D. (1897). The early development of marine annelids. J. Morph. 13, 227-326.

656 35. Woltereck, R. (1904). Beiträge zur praktischen Analyse der Polygordius-Entwicklung 657 nach dem 'Nordsee' - und dem 'Mittelmeer-typus'. I. Der für beide Typen gleich 658 verlaufende Entwicklungsabschnitt: vom Ei bis zum jüngsten Trochophora-Stadium. $659 \quad$ Arch. f. Entwicklungsmech. 18, 377-403.

660 36. Newby, W.W. (1940). The embryology of the echiuroid worm Urechis caupo, 661 Volume 16, (Philadelphia: The American Philosophical Society).

662 37. Gonzales, E.E., van der Zee, M., Dictus, W.J., and van den Biggelaar, J. (2007). 663 Brefeldin A or monensin inhibits the 3D organizer in gastropod, polyplacophoran, and 664 scaphopod molluscs. Dev Genes Evol 217, 105-118. 
665 38. Favata, M.F., Horiuchi, K.Y., Manos, E.J., Daulerio, A.J., Stradley, D.A., Feeser,

666 W.S., Van Dyk, D.E., Pitts, W.J., Earl, R.A., Hobbs, F., et al. (1998). Identification of

667

a novel inhibitor of mitogen-activated protein kinase kinase. J Biol Chem 273, 18623-

668 18632.

669

39. Martin-Duran, J.M., Passamaneck, Y.J., Martindale, M.Q., and Hejnol, A. (2016). The

670 developmental basis for the recurrent evolution of deuterostomy and protostomy. Nat

671 Ecol Evol 1, 5.

672 40. Hoodless, P.A., Pye, M., Chazaud, C., Labbe, E., Attisano, L., Rossant, J., and Wrana,

673

674

675

676

677

678

679

680

681

682

683

684

685

686

687

688

J.L. (2001). FoxH1 (Fast) functions to specify the anterior primitive streak in the mouse. Genes Dev 15, 1257-1271.

41. Pogoda, H.M., Solnica-Krezel, L., Driever, W., and Meyer, D. (2000). The zebrafish forkhead transcription factor FoxH1/Fast1 is a modulator of nodal signaling required for organizer formation. Curr Biol 10, 1041-1049.

42. Yamamoto, M., Meno, C., Sakai, Y., Shiratori, H., Mochida, K., Ikawa, Y., Saijoh, Y., and Hamada, H. (2001). The transcription factor FoxH1 (FAST) mediates Nodal signaling during anterior-posterior patterning and node formation in the mouse. Genes Dev 15, 1242-1256.

43. Watanabe, M., and Whitman, M. (1999). FAST-1 is a key maternal effector of mesoderm inducers in the early Xenopus embryo. Development 126, 5621-5634.

44. Pruitt, M.M., Letcher, E.J., Chou, H.C., Bastin, B.R., and Schneider, S.Q. (2014). Expression of the wnt gene complement in a spiral-cleaving embryo and trochophore larva. Int J Dev Biol 58, 563-573.

45. MacLean, J.A., 2nd, and Wilkinson, M.F. (2010). The Rhox genes. Reproduction 140, 195-213. 
689 46. Hubaud, A., and Pourquié, O. (2014). Signalling dynamics in vertebrate segmentation.

$690 \quad$ Nat Rev Mol Cell Biol 15, 709-721.

691 47. Andrikou, C., and Hejnol, A. (2021). FGF signaling acts on different levels of

692 mesoderm development within Spiralia. Development.

693

48. Sun, L., Tran, N., Liang, C., Tang, F., Rice, A., Schreck, R., Waltz, K., Shawver, L.K., McMahon, G., and Tang, C. (1999). Design, Synthesis, and Evaluations of Substituted 3-[(3- or 4-Carboxyethylpyrrol-2-yl)methylidenyl]indolin-2-ones as Inhibitors of VEGF, FGF, and PDGF Receptor Tyrosine Kinases. Journal of Medicinal Chemistry $42,5120-5130$.

49. Fuentealba, L.C., Eivers, E., Geissert, D., Taelman, V., and De Robertis, E.M. (2008). Asymmetric mitosis: Unequal segregation of proteins destined for degradation. Proc Natl Acad Sci U S A 105, 7732-7737.

50. Lambert, J.D., and Nagy, L.M. (2002). Asymmetric inheritance of centrosomally localized mRNAs during embryonic cleavages. Nature 420, 682-686.

51. Gonsalves, F.C., and Weisblat, D.A. (2007). MAPK regulation of maternal and zygotic Notch transcript stability in early development. Proc Natl Acad Sci U S A 104,

705 531-536.

706

52. Johnson, A.B., and Lambert, J.D. (2021). The Caudal ParaHox gene is required for hindgut development in the mollusc Tritia (a.k.a. Ilyanassa). Dev Biol 470, 1-9.

708 53. Gharbiah, M., Nakamoto, A., Johnson, A.B., Lambert, J.D., and Nagy, L.M. (2014).

709 Ilyanassa Notch signaling implicated in dynamic signaling between all three germ layers. Int J Dev Biol 58, 551-562.

711 54. Dorey, K., and Amaya, E. (2010). FGF signalling: diverse roles during early vertebrate embryogenesis. Development 137, 3731-3742. 
713 55. Green, S.A., Norris, R.P., Terasaki, M., and Lowe, C.J. (2013). FGF signaling induces

714 mesoderm in the hemichordate Saccoglossus kowalevskii. Development 140, 1024-

$715 \quad 1033$

716 56. McGregor, A.P., Pechmann, M., Schwager, E.E., and Damen, W.G. (2009). An

717 ancestral regulatory network for posterior development in arthropods. Commun Integr

$718 \quad$ Biol 2, 174-176.

719 57. Martin, B.L., and Kimelman, D. (2009). Wnt signaling and the evolution of embryonic 720 posterior development. Curr Biol 19, R215-219.

721 58. Fritzenwanker, J.H., Uhlinger, K.R., Gerhart, J., Silva, E., and Lowe, C.J. (2019).

722 Untangling posterior growth and segmentation by analyzing mechanisms of axis

723 elongation in hemichordates. Proc Natl Acad Sci U S A 116, 8403-8408.

724 59. Dictus, W.J.A.G., and Damen, P. (1997). Cell-lineage and clonal-contribution map of 725 the trochophore larva of Patella vulgata (Mollusca). Mechanisms of Development 62, $726 \quad 213-226$.

727 60. Lyons, D.C., Perry, K.J., and Henry, J.Q. (2015). Spiralian gastrulation: germ layer 728 formation, morphogenesis, and fate of the blastopore in the slipper snail Crepidula 729 fornicata. Evodevo 6, 24.

730 61. Koren, S., Walenz, B.P., Berlin, K., Miller, J.R., Bergman, N.H., and Phillippy, A.M. 731 (2017). Canu: scalable and accurate long-read assembly via adaptive k-mer weighting 732 and repeat separation. Genome Res 27, 722-736.

733 62. Roach, M.J., Schmidt, S.A., and Borneman, A.R. (2018). Purge Haplotigs: allelic 734 contig reassignment for third-gen diploid genome assemblies. BMC Bioinformatics 73519,460 . 
736 63. Dobin, A., Davis, C.A., Schlesinger, F., Drenkow, J., Zaleski, C., Jha, S., Batut, P.,

737 Chaisson, M., and Gingeras, T.R. (2013). STAR: ultrafast universal RNA-seq aligner.

$738 \quad$ Bioinformatics 29, 15-21.

739 64. Venturini, L., Caim, S., Kaithakottil, G.G., Mapleson, D.L., and Swarbreck, D. (2018).

740 Leveraging multiple transcriptome assembly methods for improved gene structure

$741 \quad$ annotation. Gigascience 7.

742 65. Stanke, M., Schoffmann, O., Morgenstern, B., and Waack, S. (2006). Gene prediction

743 in eukaryotes with a generalized hidden Markov model that uses hints from external

$744 \quad$ sources. BMC Bioinformatics 7, 62.

745 66. Haas, B.J., Delcher, A.L., Mount, S.M., Wortman, J.R., Smith, R.K., Jr., Hannick, Arabidopsis genome annotation using maximal transcript alignment assemblies. Nucleic Acids Res 31, 5654-5666.

67. Seppey, M., Manni, M., and Zdobnov, E.M. (2019). BUSCO: Assessing Genome Assembly and Annotation Completeness. Methods Mol Biol 1962, 227-245.

68. Bryant, D.M., Johnson, K., DiTommaso, T., Tickle, T., Couger, M.B., Payzin-Dogru, Cell Rep 18, 762-776.

69. Thomas, P.D., Campbell, M.J., Kejariwal, A., Mi, H., Karlak, B., Daverman, R., Diemer, K., Muruganujan, A., and Narechania, A. (2003). PANTHER: a library of protein families and subfamilies indexed by function. Genome Res 13, 2129-2141. 
improved ATAC-seq protocol reduces background and enables interrogation of frozen tissues. Nat Methods 14, 959-962.

762 71. Picelli, S., Bjorklund, A.K., Reinius, B., Sagasser, S., Winberg, G., and Sandberg, R.

763 (2014). Tn5 transposase and tagmentation procedures for massively scaled sequencing

764 projects. Genome Res 24, 2033-2040.

765

72. Martin, M. (2011). Cutadapt removes adapter sequences from high-throughput sequencing reads. 201117,3 .

767

73. Sedlazeck, F.J., Rescheneder, P., and von Haeseler, A. (2013). NextGenMap: fast and accurate read mapping in highly polymorphic genomes. Bioinformatics 29, 2790-

769 2791.

74. Li, H., Handsaker, B., Wysoker, A., Fennell, T., Ruan, J., Homer, N., Marth, G., Abecasis, G., Durbin, R., and Genome Project Data Processing, S. (2009). The Sequence Alignment/Map format and SAMtools. Bioinformatics 25, 2078-2079.

773 75. Ramirez, F., Ryan, D.P., Gruning, B., Bhardwaj, V., Kilpert, F., Richter, A.S., Heyne, S., Dundar, F., and Manke, T. (2016). deepTools2: a next generation web server for deep-sequencing data analysis. Nucleic Acids Res 44, W160-165.

776

76. Gaspar, J.M. (2018). Improved peak-calling with MACS2. bioRxiv, 496521. ChIP-Seq (MACS). Genome Biol 9, R137.

$78078 . \quad$ Li, Q., Brown, J.B., Huang, H., and Bickel, P.J. (2011). Measuring reproducibility of high-throughput experiments. The Annals of Applied Statistics 5, 1752-1779, 1728. 
784

785

786

787

788

789

790

791

792

793

794

795

796

797

798

799

800

801

802

803

804

805

806

807

determining transcription factors prime cis-regulatory elements required for macrophage and B cell identities. Mol Cell 38, 576-589.

80. Bentsen, M., Goymann, P., Schultheis, H., Klee, K., Petrova, A., Wiegandt, R., Fust, A., Preussner, J., Kuenne, C., Braun, T., et al. (2020). ATAC-seq footprinting unravels kinetics of transcription factor binding during zygotic genome activation. Nat Commun 11, 4267.

81. Quinlan, A.R., and Hall, I.M. (2010). BEDTools: a flexible suite of utilities for comparing genomic features. Bioinformatics 26, 841-842.

82. Lopez-Delisle, L., Rabbani, L., Wolff, J., Bhardwaj, V., Backofen, R., Gruning, B., Ramirez, F., and Manke, T. (2021). pyGenomeTracks: reproducible plots for multivariate genomic datasets. Bioinformatics 37, 422-423.

83. Bolger, A.M., Lohse, M., and Usadel, B. (2014). Trimmomatic: a flexible trimmer for Illumina sequence data. Bioinformatics 30, 2114-2120.

84. Bray, N.L., Pimentel, H., Melsted, P., and Pachter, L. (2016). Near-optimal probabilistic RNA-seq quantification. Nat Biotechnol 34, 525-527.

85. Love, M.I., Huber, W., and Anders, S. (2014). Moderated estimation of fold change and dispersion for RNA-seq data with DESeq2. Genome Biol 15, 550.

86. Benjamini, Y., and Hochberg, Y. (1995). Controlling the False Discovery Rate: A Practical and Powerful Approach to Multiple Testing. Journal of the Royal Statistical Society. Series B (Methodological) 57, 289-300.

87. Wickham, H. (2016). ggplot2: Elegant Graphics for Data Analysis, (Springer-Verlag New York).

88. Young, M.D., Wakefield, M.J., Smyth, G.K., and Oshlack, A. (2010). Gene ontology analysis for RNA-seq: accounting for selection bias. Genome Biol 11, R14. 
808 89. Kanehisa, M., Sato, Y., Kawashima, M., Furumichi, M., and Tanabe, M. (2016).

809 KEGG as a reference resource for gene and protein annotation. Nucleic Acids Res 44,

$810 \quad$ D457-462.

$81190 . \quad$ UniProt, C. (2021). UniProt: the universal protein knowledgebase in 2021. Nucleic

$812 \quad$ Acids Res 49, D480-D489.

813 91. Katoh, K., Rozewicki, J., and Yamada, K.D. (2019). MAFFT online service: multiple

814 sequence alignment, interactive sequence choice and visualization. Brief Bioinform

$815 \quad 20,1160-1166$.

816 92. Talavera, G., and Castresana, J. (2007). Improvement of phylogenies after removing

817 divergent and ambiguously aligned blocks from protein sequence alignments. Syst

$818 \quad$ Biol 56, 564-577.

819 93. Stamatakis, A. (2014). RAxML version 8: a tool for phylogenetic analysis and post-

820 analysis of large phylogenies. Bioinformatics 30, 1312-1313.

$82194 . \quad$ Price, M.N., Dehal, P.S., and Arkin, A.P. (2010). FastTree 2--approximately

822 maximum-likelihood trees for large alignments. PLoS One 5, e9490.

823 95. Jones, P., Binns, D., Chang, H.Y., Fraser, M., Li, W., McAnulla, C., McWilliam, H., 824 Maslen, J., Mitchell, A., Nuka, G., et al. (2014). InterProScan 5: genome-scale protein 825 function classification. Bioinformatics 30, 1236-1240. 


\section{Figures}

829 Figure 1. Di-phosphorylated ERK1/2 is enriched in the 4d cell in $O$. fusiformis. (A)

830 Spiralia is a major bilaterian lineage exhibiting spiral cleavage as the most probably

831 synapomorphy. Differently from other bilaterian lineages, body patterning and embryonic

832 organisation is controlled by a cell in the D-quadrant, which in molluscs exhibits and requires

833 active di-phosphorilated ERK1/2. Whether the molluscan condition is ancestral to Spiralia is

834 unclear. (B) O. fusiformis is a member of Palaeoannelida, the sister group to all remaining

835 annelids, and undergoes typical equal spiral cleavage $(\mathbf{C})$. The transition from the 4-cell to the

8368 -cell stage is characterised by a dextral shift of the mitotic spindle at a $45^{\circ}$ angle relative to

837 the animal-vegetal axis and generates a quartet of slightly larger animal cells (the

838 micromeres, 1q) and a quartet of vegetal cells (the macromeres, 1Q). (D) Active di-

839 phosporylated-ERK1/2 (yellow) is enriched in four animal cells $\left(1 \mathrm{q}^{111}\right)$ at 4 hours post

840 fertilisation (hpf), in a single cell (the $4 \mathrm{~d}$ micromere) at $5 \mathrm{hpf}$, and in six cells of the $2 \mathrm{a}-\mathrm{c}$

841 lineage and 4d at 6 hpf. DAPI: cyan. (E) Dynamics of cleavage and quadrant identities during

842 the onset and early division of $4 \mathrm{~d}$. The fourth micromere quartet emerges asymmetrically

843 (early $4 \mathrm{hpf}$ ), which is soon followed by the asymmetric division of one of the $3 \mathrm{q}$ micromeres

844 (arrowheads) (late $4 \mathrm{hpf}$ ). The vegetal pole is however quatri-radial at $5 \mathrm{hpf}$, with the $4 \mathrm{~d}$

845 arresting its division with respect to $4 \mathrm{a}-\mathrm{c}$, and only cleaving into MR and ML after ingression

846 at 7 hpf. F-actin (grey) shows cell boundaries and DAPI stains the nuclei. False colouring

847 indicates cell or quadrant identities. Asterisks in (C) and (D) point to the animal pole, and to

848 part of the $4 \mathrm{~d}$ progeny in $(\mathbf{E})$. Descriptions for $(\mathbf{D})$ and $(\mathbf{E})$ are based on at least 10 embryos

849 per stage, except at $7 \mathrm{hpf}(\mathrm{n}=3)$. Drawings are not to scale. Scale bars are $50 \mu \mathrm{m}$.

850

851 Figure 2. ERK1/2 signalling controls axial polarity in O. fusiformis. (A) ERK1/2

852 signalling cascade responds to cell-cell communication and involves the di-phosphorylation 
853 of ERK1/2 (di-P-ERK1/2) via MEK. Brefeldin A (BFA), a protein trafficking inhibitor, and

854 U0126, a specific MEK inhibitor, can block ERK1/2 di-phosphorylation. (B) Immunostaining

855 against di-P-ERK1/2. BFA and U0126 treatments from 0.5 hours post fertilisation (hpf) to 5

856 hpf prevent di-P-ERK1/2 enrichment in the 4d cell at 5 hpf. (C) BFA and U0126 treatment

857 ( $0.5 \mathrm{hpf}$ to $5 \mathrm{hpf}$ ) result in anteroventrally radialised larvae that fail to form posterior

858 structures (e.g., hindgut, mesodermal bands and chaetae). Main panels are lateral z-stack

859 projections of confocal images and insets in treated embryos are ventral views. Cilia is

860 labelled with anti-acetylated tubulin. (D) Whole mount in situ hybridisation on control and

861 U0126 treated ( 0.5 to $5 \mathrm{hpf}$ ) larvae. Treated radial larvae show reduced apical markers

862 (six3/6, and syt-1 in the inset), radially expanded anterior markers ( $g s c)$, and absent posterior

863 endomesodermal ( $c d x$, twist) but not a midgut gene (GATA4/5/6b). (E) Schematic

864 representation of drug treatment windows and observed larval phenotypes (WT, wild type; R,

865 radial). (F) Morphological analysis of the compressed larval phenotype obtained after BFA

866 treatment from 0.5 hpf until 3-4 hpf. Treated larvae have a reduced apical organ and apical

867 tuft (first row) and reduced expression of apical gene markers (six3/6, and syt-1 in the inset),

868 but other tissues are not affected. The larval blastocele is somehow obliterated. Asterisks

869 point to the apical pole. Schematic drawings are not to scale. ao: apical organ; an: anus; ch:

870 chaetae; mg: midgut; mo: mouth; pt: prototroch. Scale bars are $50 \mu \mathrm{m}$.

871

872 Figure 3. ERK1/2 signalling activates posterodorsal and mesodermal genes. (A)

873 Experimental design for RNA-seq collections. Solid colour bars show period of control

874 (DMSO), BFA or U0126 treatments. Red circles show sample collection, at the coeloblastula

875 stage (5.5 hpf) and at the larval stage (24 hpf). (B) Number of differentially expressed (DE)

876 genes in different conditions and comparisons, where red bars are downregulated genes and

877 blue bars are upregulated genes. (C) Volcano plots for BFA and U0126 treated coeloblastula. 
878 Red dots show DE genes, with candidate genes labelled for each comparison. (D) Temporal

879 and spatial time course of expression of candidate genes affected by $4 \mathrm{~d}$ misspecification and

880 associated with apical/anterior, chaetae/posterior, hindgut or mesoderm development. The

881 heatmap depicts the normalised z-score value of expression for each gene throughout

882 development, with red blocks indicating high levels of expression and blue blocks indicating

883 low levels of expression. The horizontal dotted line highlights the timing of $4 \mathrm{~d}$ specification.

884 On the right, whole mount in situ hybridisation of a subset of these 22 candidate genes at the

885 coeloblastula (5 hpf), gastrula (9 hpf) and larval stages (24 hpf). (E) Only three of the

886 candidate genes ( $c d x$, foxH and $f e r 3)$ are downregulated in all stages and treatment

887 comparisons. Validation via in situ hybridisation demonstrates the expression is lost after

888 drug treatment. Pictures for $c d x$ at the coeloblastula and larva in (D) and (E) are the same.

889 Asterisks point to the animal/apical pole, and arrowheads to the domains of expression.

890 Schematic drawings are not to scale. bp: blastopore; mo: mouth.

891

892 Figure 4. ERK1/2 signalling specifies and patterns the D-quadrant. (A) Whole mount in 893 situ hybridisation of a subset of genes (ERK1/2-dependent and independent) patterning the

894 vegetal pole at (5 hpf) and after ( $5.5 \mathrm{hpf}) 4 \mathrm{~d}$ specification. The row below is a schematic 895 representation of the vegetal pole depicting gene expression (in green) at $5.5 \mathrm{hpf}$. (B) The

896 ParaHox gene $c d x$ (yellow) is expressed in the $4 \mathrm{~d}$ cell at the coeloblastula stage and in the

897 progeny of MR and ML at the gastrula stage, corroborating the endodermal sublineage of 4d.

898 (C) The Notch ligand delta (yellow) is expressed in 4d, multiple cells from the D-quadrant, 899 and a few animal micromeres from the other three quadrants at the coeloblastula stage. In (B,

900 C) Nuclei (DAPI) are shown in cyan and cell boundaries (acetylated tubulin) in magenta.

901 Asterisks point to the animal pole. (D) Distribution of known bound transcription factors in 902 open chromatin regions around $c d x$ (upper part) and delta (lower part) loci at 5 hpf. Dark and 
903 light blue depict the two ATAC-seq replicates. (E) Number of cellular contacts for each of the

$9044 \mathrm{q}$ micromeres at $5.5 \mathrm{hpf}$ based on the analysis of eight embryos. While $4 \mathrm{a}^{1}-4 \mathrm{c}^{2}$ establish 8

905 direct cell contacts, $4 \mathrm{~d}$ touches 13 cells of the D and C quadrants. (F) Inhibition of ERK1/2

906 di-phosphorylation by U0126 prevents D-quadrant specification, as shown by the expansion

907 of the anterior marker gsc and loss of the posterior marker AP2. The $4 \mathrm{~d}$ micromere is no

908 longer recognised, as all $4 \mathrm{q}$ micromeres cleave at the same time (asterisks). However, $g s c$ is

909 lost in BFA treated embryos, and thus needs of inductive signals. Gene expression is shown in

910 yellow, nuclei (DAPI) in cyan and cell boundaries (acetylated tubulin) in magenta or grey. In

911 all panels, arrowheads point to expression domains. Schematic drawings are not to scale. gp:

912 gastral plate.

913

914 Figure 5. FGFR signalling regulates ERK1/2 di-phosphorylation in 4d. (A) The drug

915 SU5402 specifically inhibits receptors tyrosine kinase of the type FGF/VEGF, which can

916 signal through the ERK1/2 signalling cascade. (B) Whole mount immunohistochemistry

917 against di-phosphorylated ERK1/2 (di-P-ERK1/2). SU5402 treated embryos lose ERK1/2

918 enrichment in a vegetal micromere at 5 hours post fertilisation (hpf). (C) Schematic

919 representation of drug treatment windows and observed larval phenotypes (WT, wild type).

920 (D) Morphological and molecular patterning analysis of SU5402 treated embryos (4 to 6 hpf)

921 fixed at $24 \mathrm{hpf}$. The first column shows diagrams of lateral views of the wild type in DMSO

922 controls and radial larvae in treated embryos. Second and third columns show larvae labelled

923 with DAPI (cyan) and F-actin (magenta) plus acetylated tubulin staining (yellow),

924 respectively. Fourth column onwards are whole mount in situ hybridisations. SU5402

925 treatment from $4 \mathrm{hpf}$ to $6 \mathrm{hpf}$ results in phenocopies of U0126 and BFA radial larvae. Insets

926 are ventral views. Asterisks point to the apical pole. Schematic drawings are not to scale. ao:

927 apical organ; an: anus; ch: chaetae; mg: midgut; mo: mouth; pt: prototroch. 
929 Figure 6. ERK1/2 activity in the D-quadrant embryonic organiser is an ancestral

930 spiralian trait. (A) Schematic model of FGFR/ERK1/2 activity in the $4 \mathrm{~d}$ micromere during

931 late spiral cleavage in $O$. fusiformis. At 4 hours post fertilisation (hpf), di-phosphorylated

932 ERK1/2 (di-P-ERK1/2) is enriched in the apical most micromeres $\left(1 \mathrm{q}^{111}\right)$. At $5 \mathrm{hpf}$, an

933 unidentified signal activates FGF receptor (FGFR) which di-phosphorylates ERK1/2 in the 4d

934 micromere. This activates $c d x$ and delta in $4 \mathrm{~d}$ (becoming the endomesodermal precursor) and

935 correlates with $4 \mathrm{~d}$ arresting its cell cycle progression, as $4 \mathrm{a}-4 \mathrm{c}$ cleave. ERK1/2 activation in

$9364 \mathrm{~d}$ is required to limit an anteriorising signal (Signal $\mathrm{X}$ ) to the A-C quadrants, which activates

937 gsc expression in these quadrants. In addition, ERK1/2 activity in 4d induces the expression

938 of a battery of genes in the D-quadrant and part of the C-quadrant that probably induce lateral

939 mesodermal and posterodorsal fates, ultimately controlling axial elongation. This inductive

940 activity might happen via the Notch pathway. (B) The similarities in ERK1/2 activation

941 patterns between $O$. fusiformis and other molluscan embryos suggest this is a shared ancestral

942 trait to spiral cleavage. More broadly, ERK1/2 activity induces the expression of a set of

943 genes and signalling pathways that are repeatedly involved in posterior specification and

944 anteroposterior growth in bilaterian embryos. Drawings are not to scale.

945

946 


\section{Supplemental Information}

948 Figure S1. ERK1/2 activity during $O$. fusiformis early embryogenesis. (A) Maximum

949 likelihood orthology assignment for ERK1/2, using ERK5 as outgroup. Only bootstrap values

950 for major nodes are shown. (B) DEseq2 normalised expression of erk1/2 during $O$. fusiformis

951 development, from the active oocyte to the mitraria larval stage. The time of the specification

952 of $4 \mathrm{~d}$ is highlighted with a schematic drawing. Coloured dots indicate values of expression for

953 each replicate and the dotted red line shows the mean value of expression. (C) Whole mount

954 immunohistochemistry against di-phosphorylated-ERK1/2 (dark orange) during spiral

955 cleavage in $O$. fusiformis. ERK1/2 is active at low levels during early divisions, and later

956 enriched in the four $1 \mathrm{q}^{111}$ animal micromeres at 4 hours post fertilisation (hpf), the $4 \mathrm{~d}$

957 micromere at the time of its specification ( $5 \mathrm{hpf})$ and finally in six vegetal cells forming a

958 bilaterally symmetrical pattern (arrowheads) at 6 hpf. Insets in (C) show either nuclear

959 staining (blue) or lateral views. Asterisks point to the animal pole.

960

961 Figure S2. The effect of BFA and U0126 treatment in $O$. fusiformis development. (A)

962 Percentage of coeloblastula showing di-phosphorylated ERK1/2 (di-P-ERK1/2) enrichment in

963 the $4 \mathrm{~d}$ micromere after 0.5 to 5 hours post fertilisation (hpf) treatment with brefeldin A

964 (BFA), U0126 or DMSO (control). (B) Percentage of radial phenotypes obtained when

965 treating embryos from 0.5 to $5 \mathrm{hpf}$ with a range of BFA and U0126 concentrations. (C)

966 Whole mount in situ hybridisation of apical (six3/6), oral $(g s c)$, posterior/hindgut $(c d x)$,

967 midgut (GATA4/5/6b) and trunk mesodermal (twist) gene markers in control and treated

968 larvae. Radial phenotypes have a reduction of the apical marker, radial expansion of an oral

969 gene, loss of posterior structures (chaetae) and posterior/trunk mesoderm gene expression and

970 retain the expression of a midgut gene. Radial larvae treated with U0126 from the 2-cell stage

971 to $3 \mathrm{hpf}$ show scattered twist expression in some embryos (arrowheads). The compressed 
972 larval phenotype after BFA treatment from 0.5 to $4 \mathrm{hpf}$ have a reduced apical organ and

973 apical tuft (first row) and reduced expression of apical markers (six3/6), but otherwise normal

974 morphology besides an obliterated internal blastocele. Asterisks indicate the apical pole and

975 arrowheads point to expression domains. Insets are ventral views. mo: mouth.

976

977 Figure S3. Differential expression analyses in BFA and U0126 treated embryos. (A)

978 Hierarchical pairwise correlation matrix between brefeldin A (BFA) and U0126 treated and

979 control samples. (B) Principal component analysis (PCA) plot for BFA (left) and U0126

980 (right) RNA-seq analyses. (C) Volcano plots for BFA and U0126 treated larva. Red dots

981 show differentially expressed (DE) genes and analysed candidate genes are labelled in each

982 comparison. (D) Venn diagrams showing the number of up- (top) and downregulated

983 (bottom) DE genes shared between different conditions. (E) Ten most overrepresented KEGG

984 pathways in each of the four conditions analysed by RNA-seq. The size of the coloured dots

985 is proportional to the number of overrepresented sub-categories nested into a given KEGG

986 pathway.

987

988

Figure S4. Validation of the differential expression analyses. (A-G) Whole mount in situ

989

hybridisation of control and treated embryos showing in addition a wild type gastrula stage to

990 reconstruct the time course of expression of each gene. In all cases, the expression patterns

991 disappear in BFA and U0126 treated embryos. Insets are ventral views. Asterisk point to the

992 animal/apical pole. Arrowheads point to $4 \mathrm{~d}$ in (A) and to the apical organ in (F). bp:

993 blastopore; gp: gastral plate; mo: mouth.

994

995 Figure S5. Genes patterning the D-quadrant after the specification of the 4d-organiser

996 cell. (A) Schematic drawing of the cellular arrangements and cell nomenclature of the vegetal 
997 pole just after the specification of the $4 \mathrm{~d}$ micromere in $O$. fusiformis embryo at 5.5 hours post

998 fertilisation (hpf). Different colours depict different quadrants. (B) Time course of expression

999 of $c d x$, foxH and fer3 via whole mount in situ hybridisation from 6 hpf to 18 hpf during $O$.

1000 fusiformis embryogenesis. While $c d x$ is expressed in the cells forming the hindgut, foxH

1001 appears to upregulate at the earliest stages of cells giving rise to mesodermal derivatives. The

1002 gene fer3 is expressed in two posterior most cells of unknown function in the gastrula and

1003 larva. Insets are lateral views. (C) The protein domain architecture of the Notch ligand

1004 DELTA in O. fusiformis in comparisons with the domain structures of the Delta orthologs in

1005 D. melanogaster and H. sapiens. (D-G) Maximum likelihood orthology assignments of

1006 DELTA, FOXH, WNT ligands, and RHOX in O. fusiformis. Only bootstrap values supporting

1007 each major clade are shown. bp: blastopore; mo: mouth.

1008

1009 Figure S6. The FGF pathway and FGFR activity during 4d specification in $\boldsymbol{O}$.

1010 fusiformis. (A) Maximum likelihood orthology assignment of fibroblast growth factor

1011 receptor (FGFR) and vascular endothelial growth factor receptor (VEGFR). Only bootstrap

1012 values for key nodes are shown. (B) Multiple protein alignment between $O$. fusiformis and $H$.

1013 sapiens FGFR and VEGFR orthologs indicating conserved residues for the interaction of the

1014 drug SU5402 with these receptors. (C) Temporal time course of expression of members of the

1015 FGF and VEGF pathways in $O$. fusiformis. The heatmap depicts the normalised z-score value

1016 of expression for each gene, with red blocks indicating high levels of expression and blue

1017 blocks indicating low levels of expression. The horizontal dotted line highlights the

1018 specification of the $4 \mathrm{~d}$ micromere. (D) Whole mount in situ hybridisation for FGFR and

1019 VEGFR at the coeloblastula stage of $O$. fusiformis. FGFR is expressed weakly at the gastral

1020 plate at the time of $4 d$-instructed posterior re-pattering. (E) Proportion of treated and control

1021 embryos exhibiting di-phosphorylated ERK1/2 enrichment in the $4 \mathrm{~d}$ cell at $5 \mathrm{hpf}$ after 
1022 SU5402 drug treatment from 0.5 to 5 hours post fertilisation (hpf). (F) Morphological and

1023 molecular characterisation of SU5402 treated embryos fixed at the larval stage. The first

1024 column shows diagrams of lateral views of the wild type, compressed and radial phenotypes.

1025 From the second column onwards, whole mount in situ hybridisations of control and SU5402

1026 treated larvae. The compressed larvae are phenocopies of brefeldin A (BFA) compressed

1027 larvae, with reduced apical organ and apical tuft (second row) and reduced expression of

1028 apical markers (six3/6), but other tissues are not affected. Radial larvae are phenocopies of

1029 BFA and U0126 radial larvae with reduction of apical markers (six3/6), radial expansion of

1030 oral genes $(\mathrm{gsc})$, loss of posterior structures (chaetae) and expression of posterior

1031 endomesodermal markers ( $c d x$, twist), but normal expression of an endodermal gene

$1032(G A T A 4 / 5 / 6 b)$. Insets are ventral views. Asterisks point to the animal/apical pole. ao: apical

1033 organ; an: anus; ch: chaetae; gp: gastral plate; mg: midgut; mo: mouth; pt: prototroch. Scale

1034 bars are $50 \mu \mathrm{m}$. 
bioRxiv preprint doi: https://doi.org/10.1101/2021.05.26.445819; this version posted May 27, 2021. The copyright holder for this preprint (which

was not certified by peer review) is the author/funder, who has granted bioRxiv a license to display the preprint in perpetuity. It is made available under aCC-BY-NC 4.0 International license.

\section{Supplementary Tables and Files}

1036 Table S1. Di-P-ERK1/2 activation in spiralians.

1037 Table S2. Di-P-ERK1/2 activation at $5 \mathrm{hpf}$ in control, BFA and U0126 treated embryos.

1038 Table S3. Larval phenotypes in control, BFA and U0126 treatments.

1039 Table S4. Larval phenotypes at different concentrations of BFA and U0126.

1040 Table S5. Larval phenotypes during staggered time windows of BFA and U0126 treatments.

1041 Table S6. List of all differentially expressed genes at $5.5 \mathrm{hpf}$ and $24 \mathrm{hpf}$.

1042 Table S7. List of enriched gene ontology terms.

1043 Table S8. List of enriched KEGG pathways.

1044 Table S9. List of candidate genes and their gene IDs in differential gene expression analyses.

1045 File S1. Multiple protein alignments 
Embryonic organisers \& body patterning

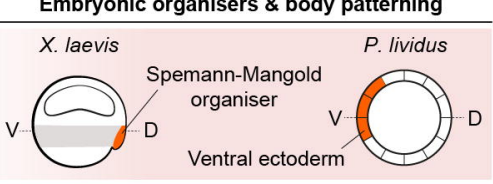

Deuterostomia

Vertebrates, sea urchin

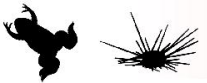

Ecdysozoa

Nematode, fly

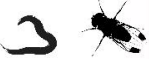

C. elegans

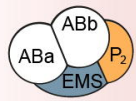

D. melanogaster

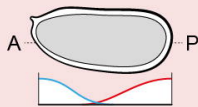

Gnathifera

Platyhelminthes

(8) ?

Spiral cleavage

$>32$-cell stage

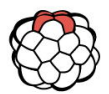

$4 \mathrm{hpf}$

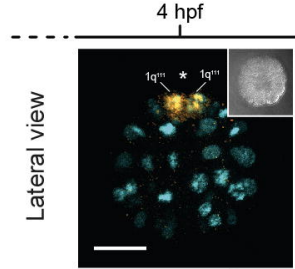

Lophophorata $\checkmark$

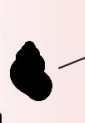
D organiser

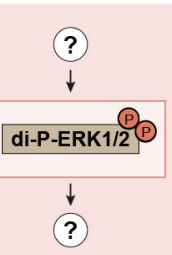

E
Annelida Mollusca Nemertea

Coeloblastula

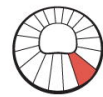

5 hpf

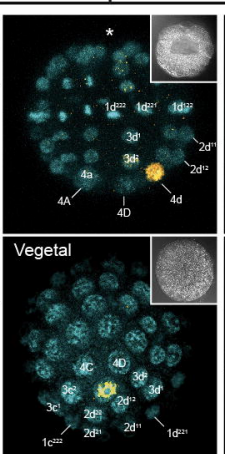

B
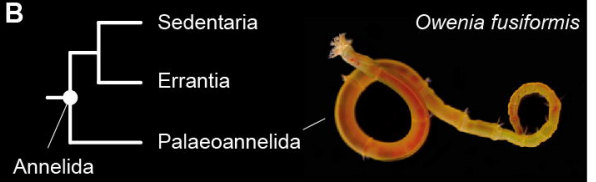

C
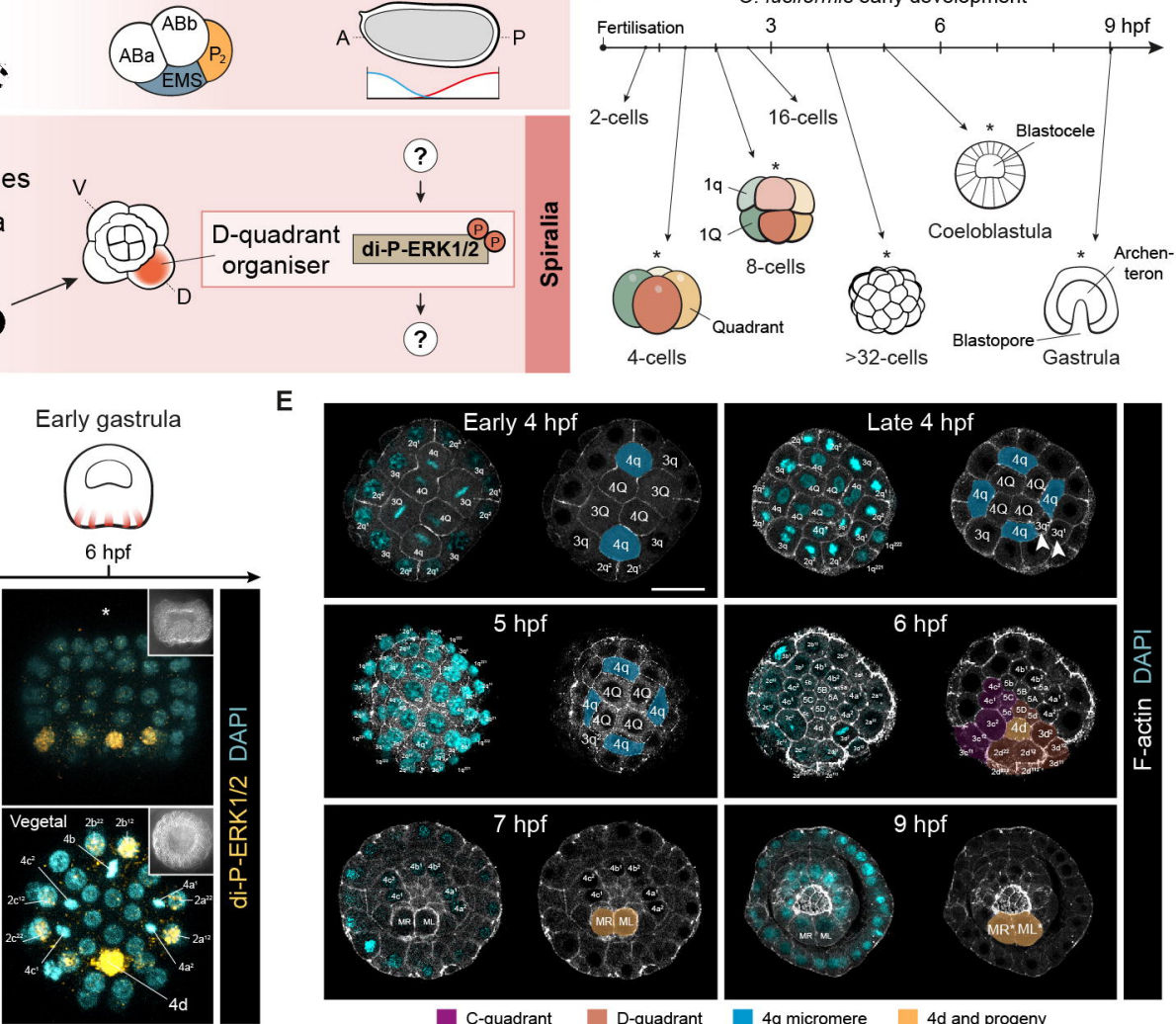

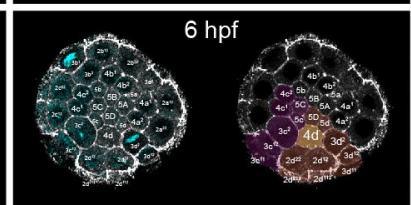

$9 \mathrm{hpf}$

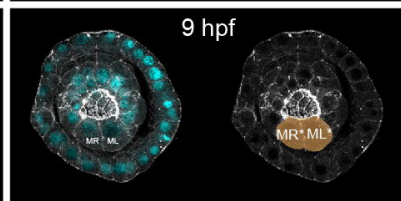

C-quadrant

$4 q$ micromere

$4 d$ and progeny

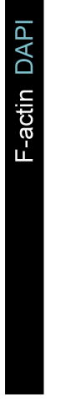


A

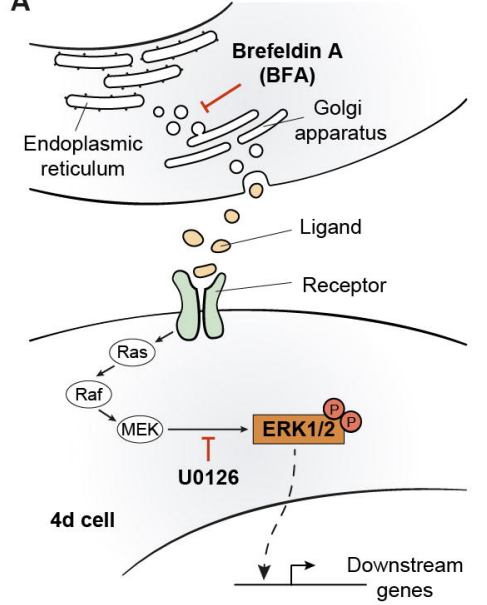

E

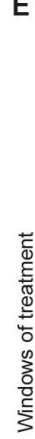

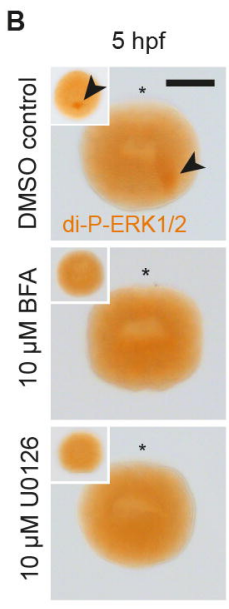

Organiser specification

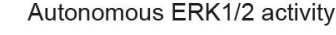

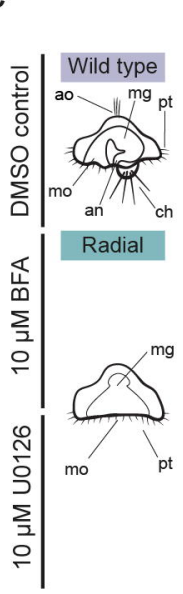
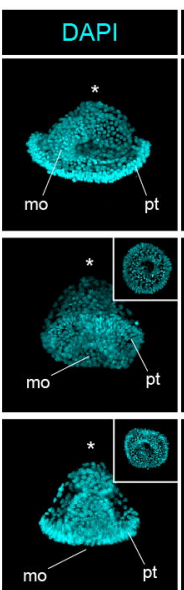

mo
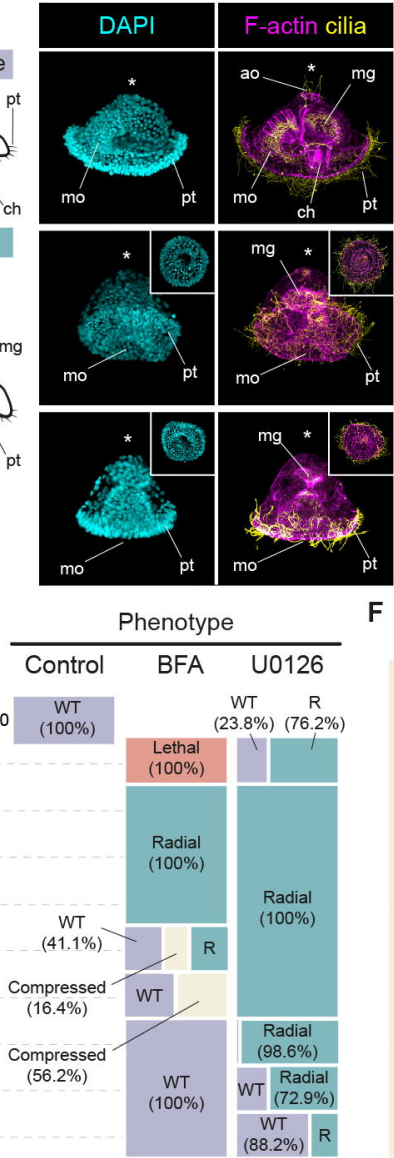

F
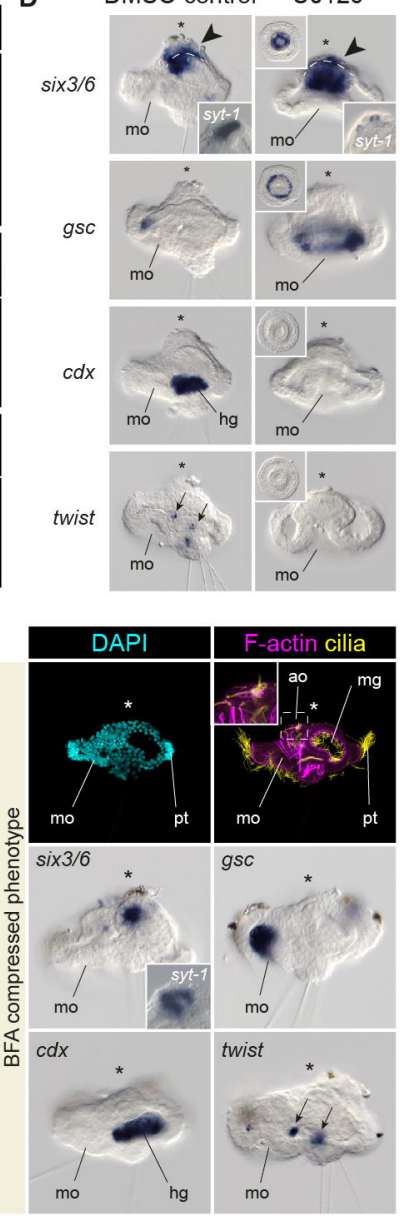


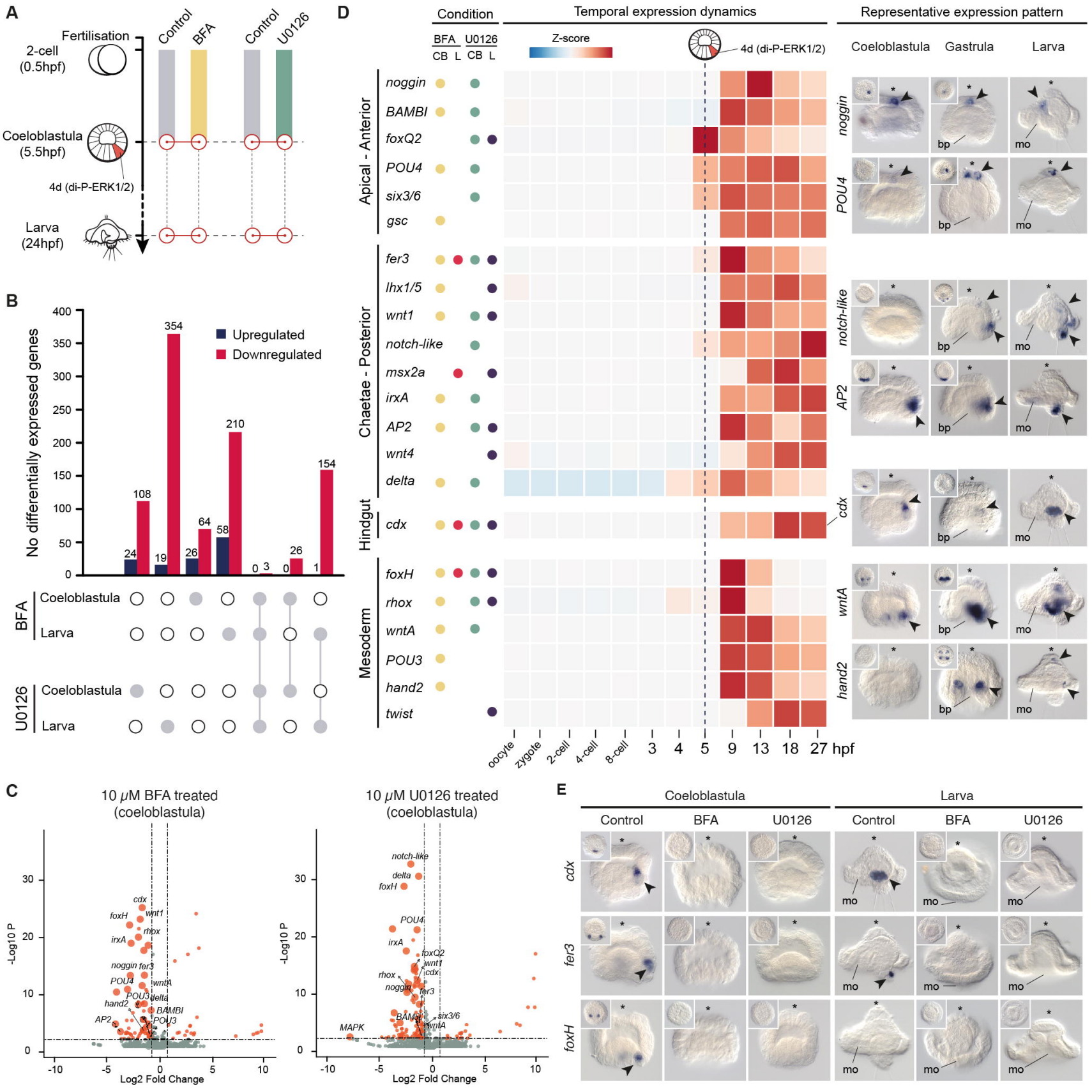



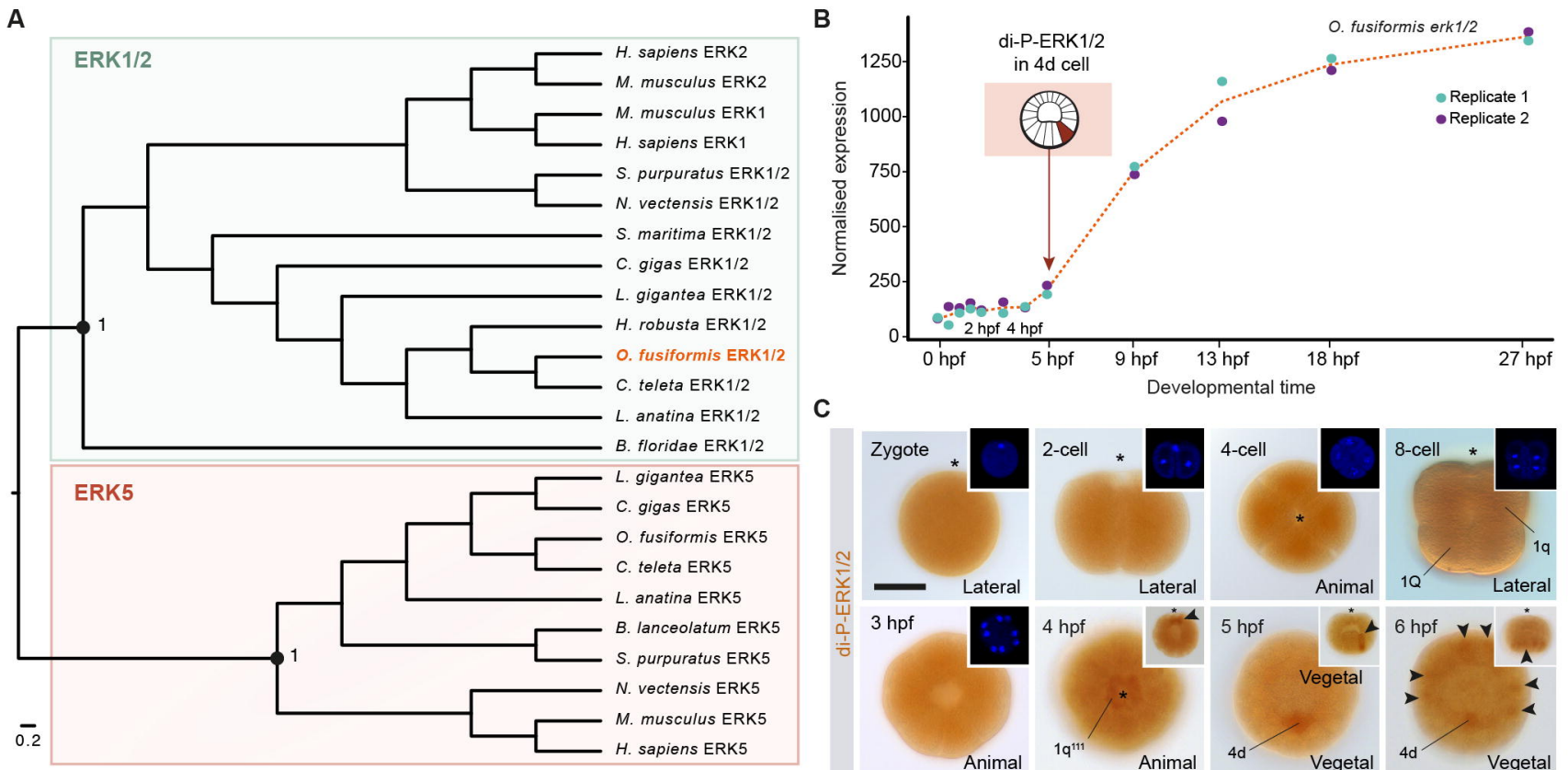

C

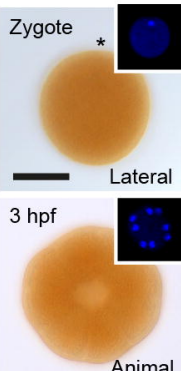

2-cell

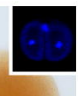

4-cell

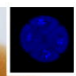

8-cell

Animal
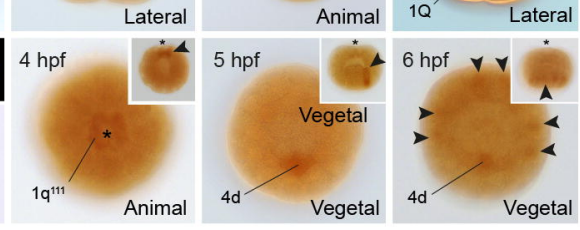
A Coeloblastula Control - Coeloblastula $10 \mu \mathrm{M}$ BFA - Larva Control - Larva $10 \mu \mathrm{M}$ BFA

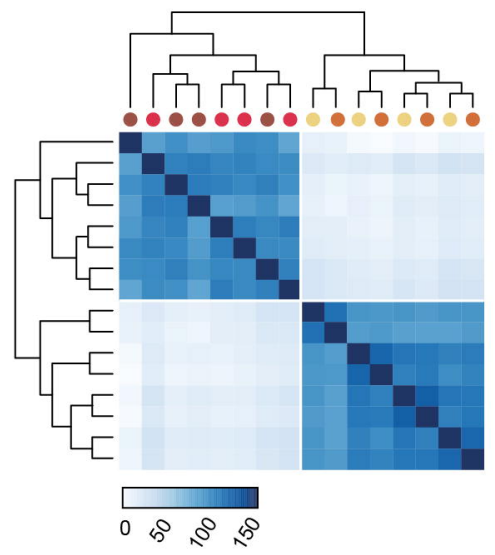

B

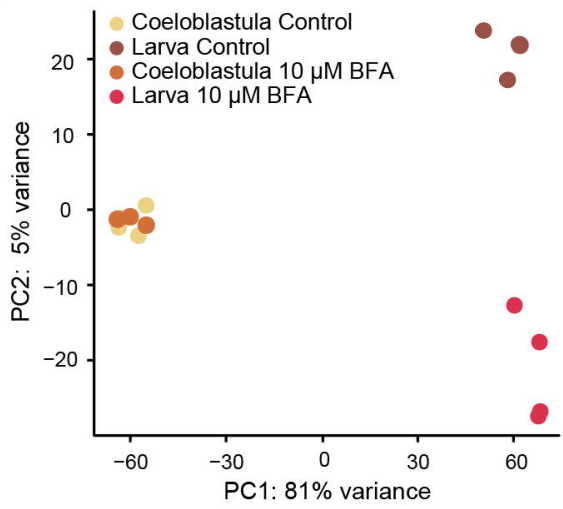

C

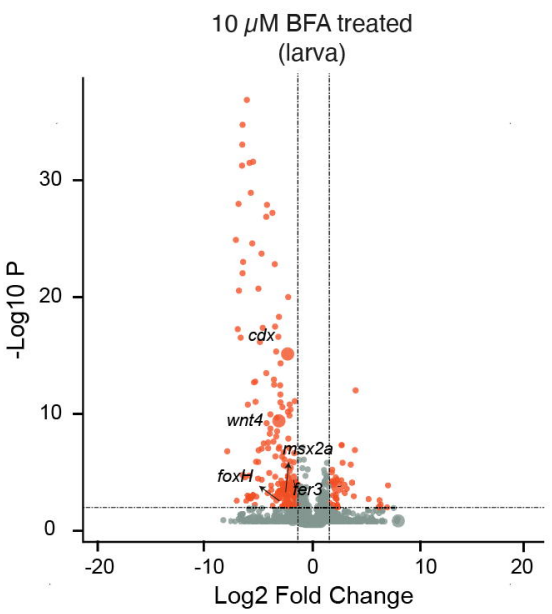

Coeloblastula Control Coeloblastula 10 HM U0126

- Larva Control - Larva $10 \mu \mathrm{M}$ U0126
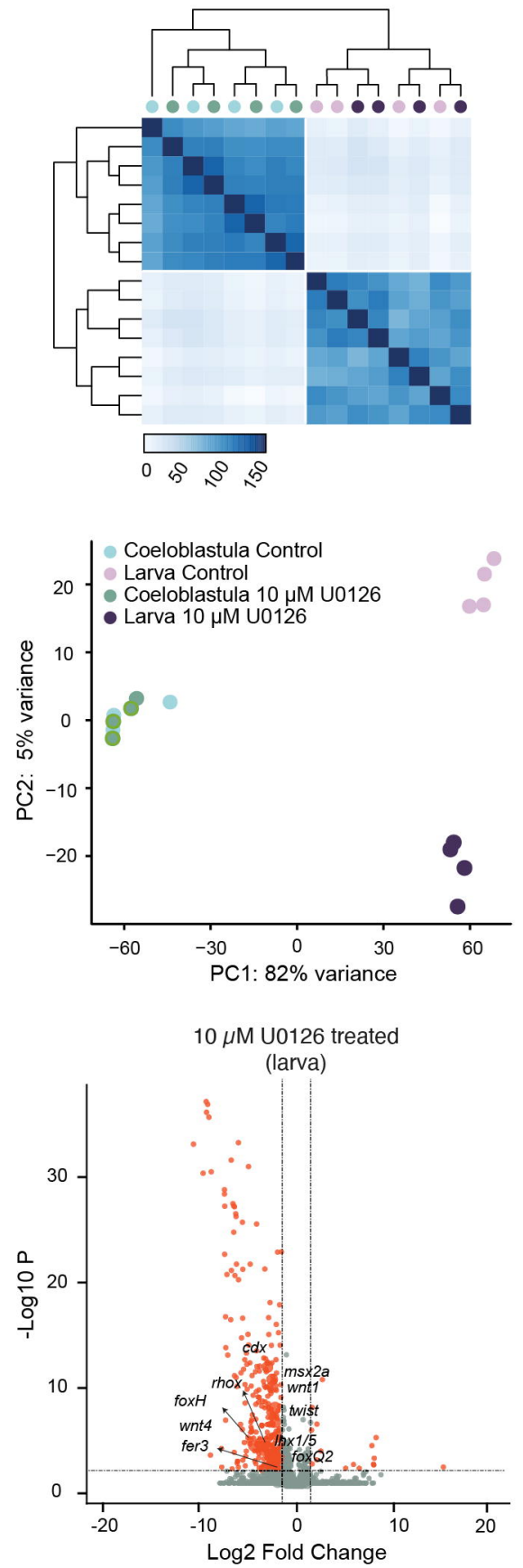

\section{Upregulated}

U0126 BFA

Larva Coeloblastula Coeloblastula Larva

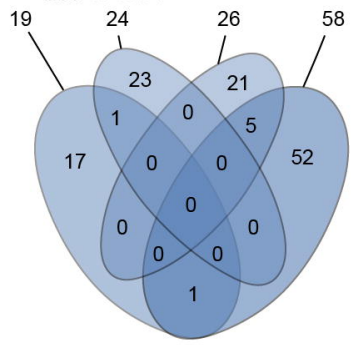

\section{Downregulated}

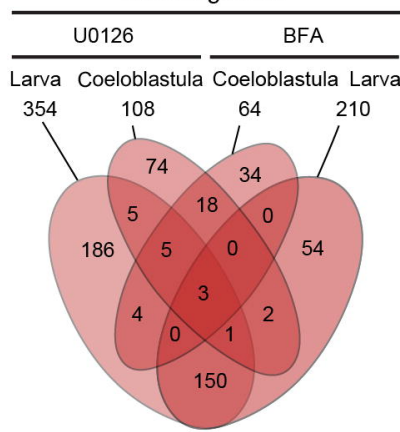

E

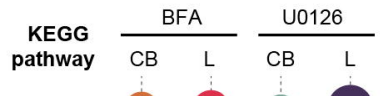

mTOR

cGMP-PKG

cAMP

Wnt

TGF-beta

Ras

Phospholipase D

PI3K-Akt

MAPK

Hippo

Number of over-represented sub-categories

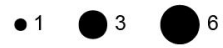




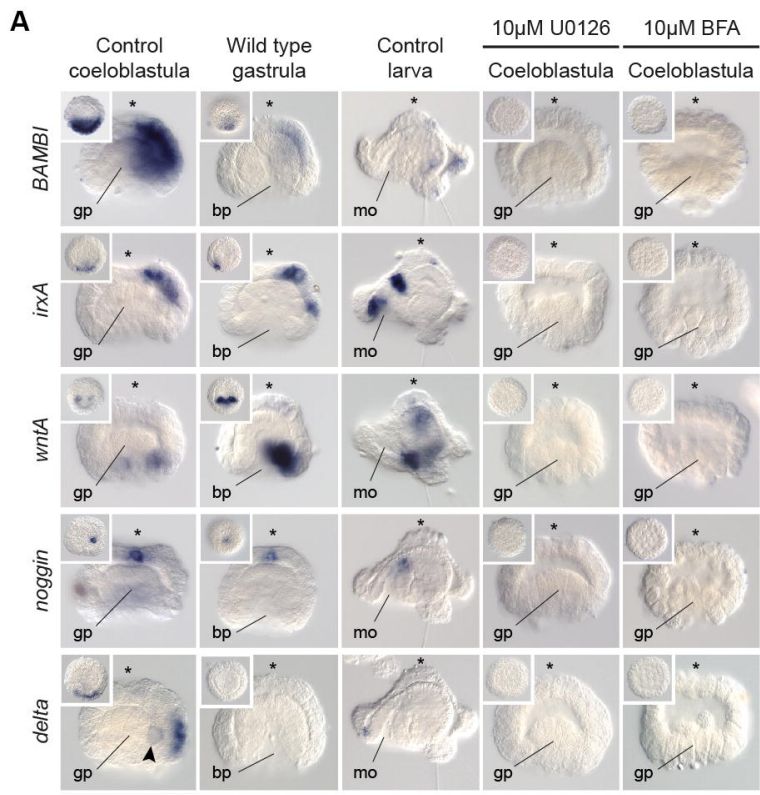

B

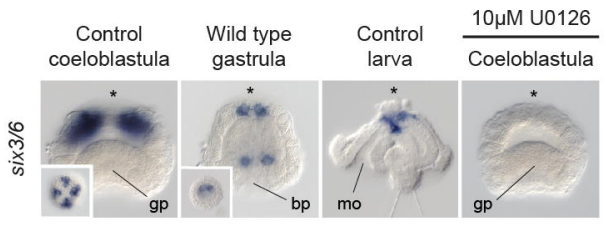

D

E

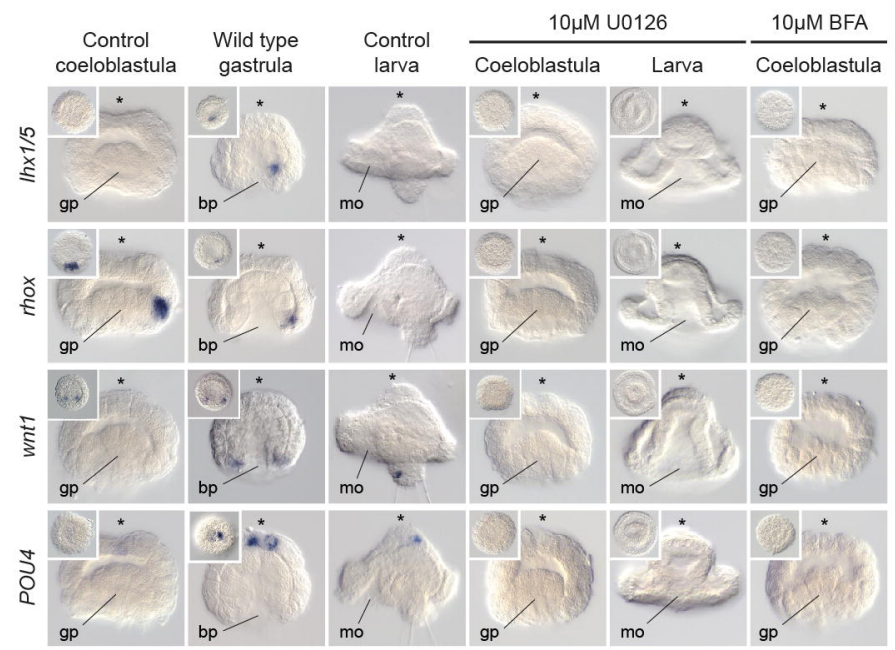

C

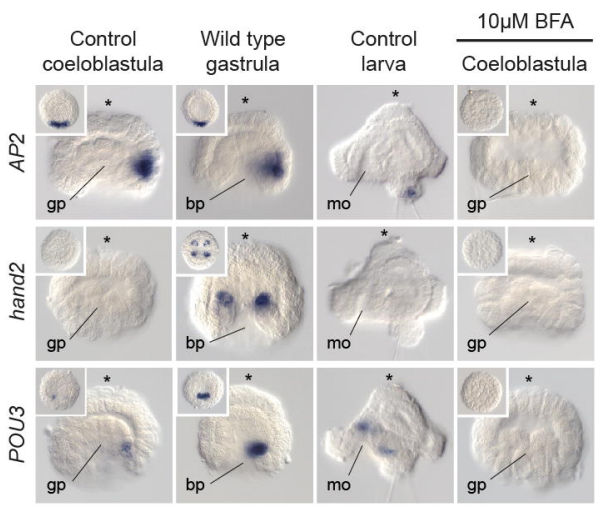

F

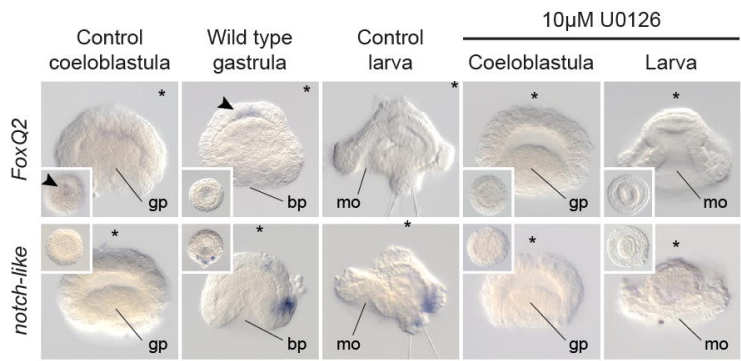

G

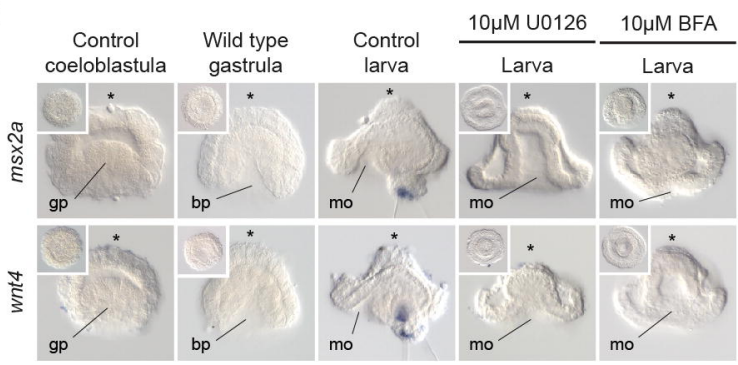




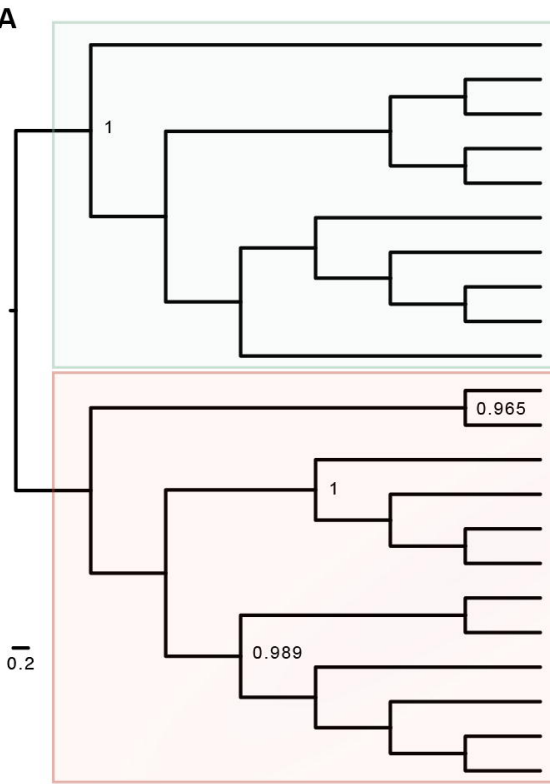

purpuratus FGFR

H. sapiens FGFR4

H. sapiens FGFR3

H. sapiens FGFR2

O. fusiformis VEGFR

D. melanogaster Q95P10

H. sapiens PGFRB

$X$. laevis PGFRA

G. gallus PGFRA

H. sapiens PGFRA

H. sapiens VGFR3

D. rerio VGFR3

D. rerio VGFR2

D

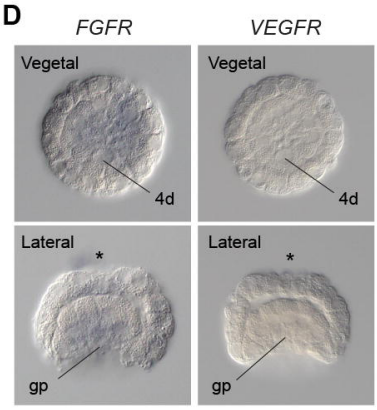

\section{$\mathrm{E}$}
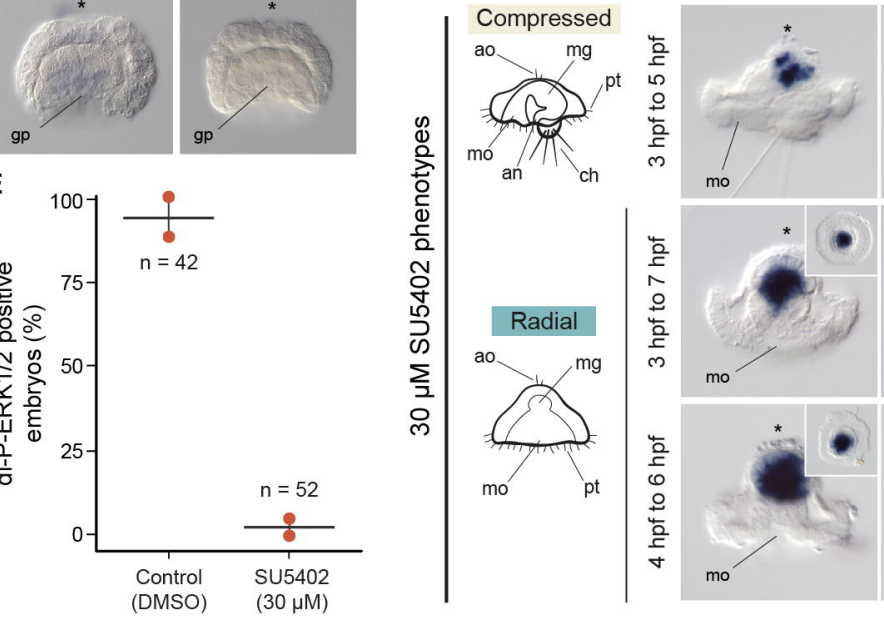

H. sapiens VGFR1

D. rerio VGFR4

H. sapiens VGFR2

VEGFR/

PDGFR

$\mathbf{F}$
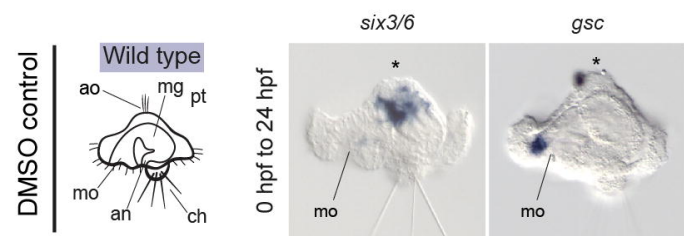

VEGF

荷网

VEGFR

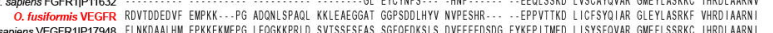

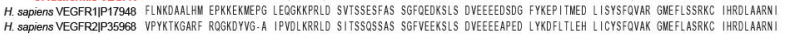

$$
220 \quad 240 \quad 260 \quad 280
$$

300

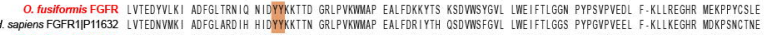
O. Fusiformis VEGFR IVAEDRVUKI ADFGLAKDVY KYEEYVKKGA GALPIKMLLLL ESL THKVFSY KTDWWAYGIL LYETFSLGGT PYPGVDLDAT FI EKLKNGYR NEKPQFASFQ

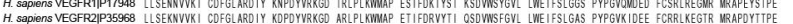

320

\section{SU5402 interaction aa}

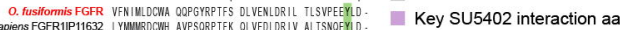

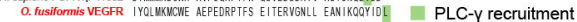

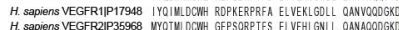

PLC-y recruitment

C

(di -4 (di-P-ERK1/2)

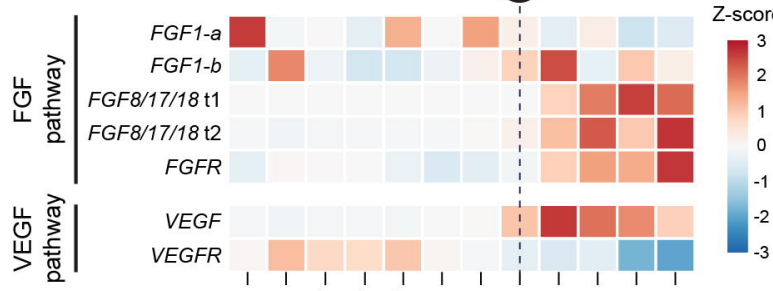

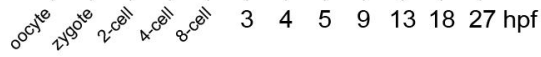
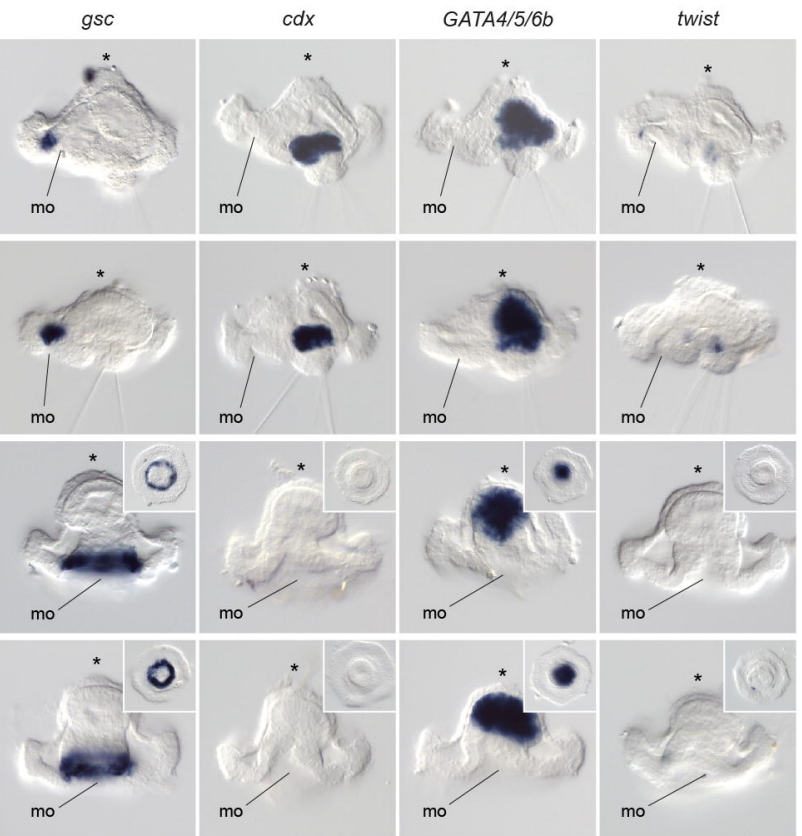